Revue des patrimoines

\title{
Forme des territoires communaux et structure du bâti dans les cantons de Vaud et de Neuchâtel
}

\section{Daniel Glauser}

\section{(2) OpenEdition}

Journals

Édition électronique

URL : http://journals.openedition.org/insitu/2608

DOI : 10.4000/insitu.2608

ISSN : 1630-7305

\section{Éditeur}

Ministère de la culture

Référence électronique

Daniel Glauser, «Forme des territoires communaux et structure du bâti dans les cantons de Vaud et de Neuchâtel », In Situ [En ligne], 7 | 2006, mis en ligne le 18 avril 2012, consulté le 19 avril 2019. URL http://journals.openedition.org/insitu/2608 ; DOI : 10.4000/insitu.2608

Ce document a été généré automatiquement le 19 avril 2019

\section{(c) (i) (9)}

In Situ Revues des patrimoines est mis à disposition selon les termes de la licence Creative Commons Attribution - Pas d'Utilisation Commerciale - Pas de Modification 4.0 International. 


\title{
Forme des territoires communaux et structure du bâti dans les cantons de Vaud et de Neuchâtel
}

\author{
Daniel Glauser
}

1 L'analyse des paysages construits des cantons de Vaud et de Neuchâtel révèle, entre Jura et Préalpes, une grande diversité des terroirs et des formes de colonisation. Leur compréhension se fonde sur la lecture des cartes anciennes et actuelles ainsi que sur les recensements architecturaux entrepris par les cantons. Ces recherches bénéficient de l'appui financier du Fonds national suisse pour la recherche scientifique et de celui des cantons concernés ; elles sont publiées par la Société suisse des traditions populaires dans

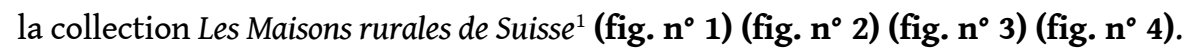




\section{Figure 1}

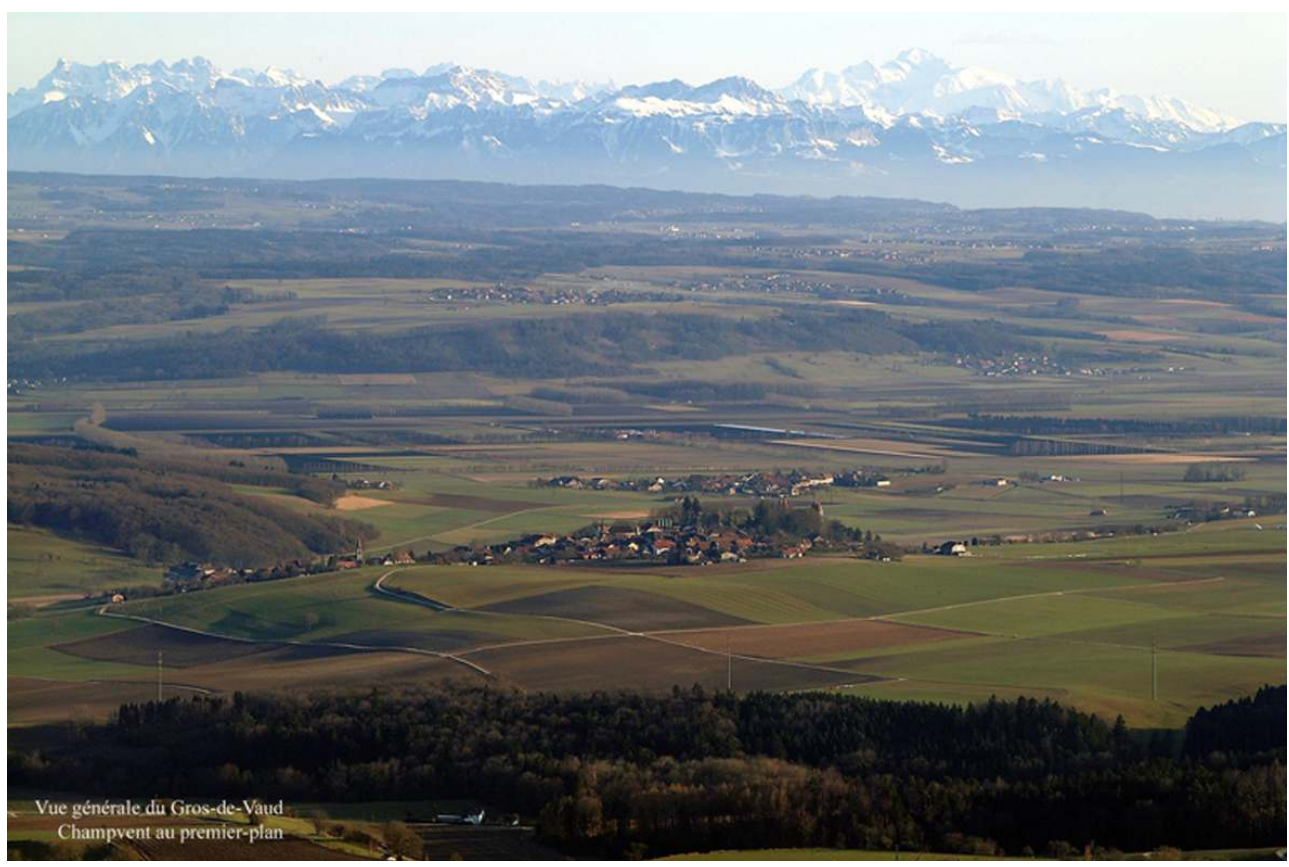

Le Gros-de-Vaud vu du Jura avec la chaîne des Alpes et le Mont-Blanc en arrière-plan Phot. D. Glauser @ D. Glauser

\section{Figure 2}

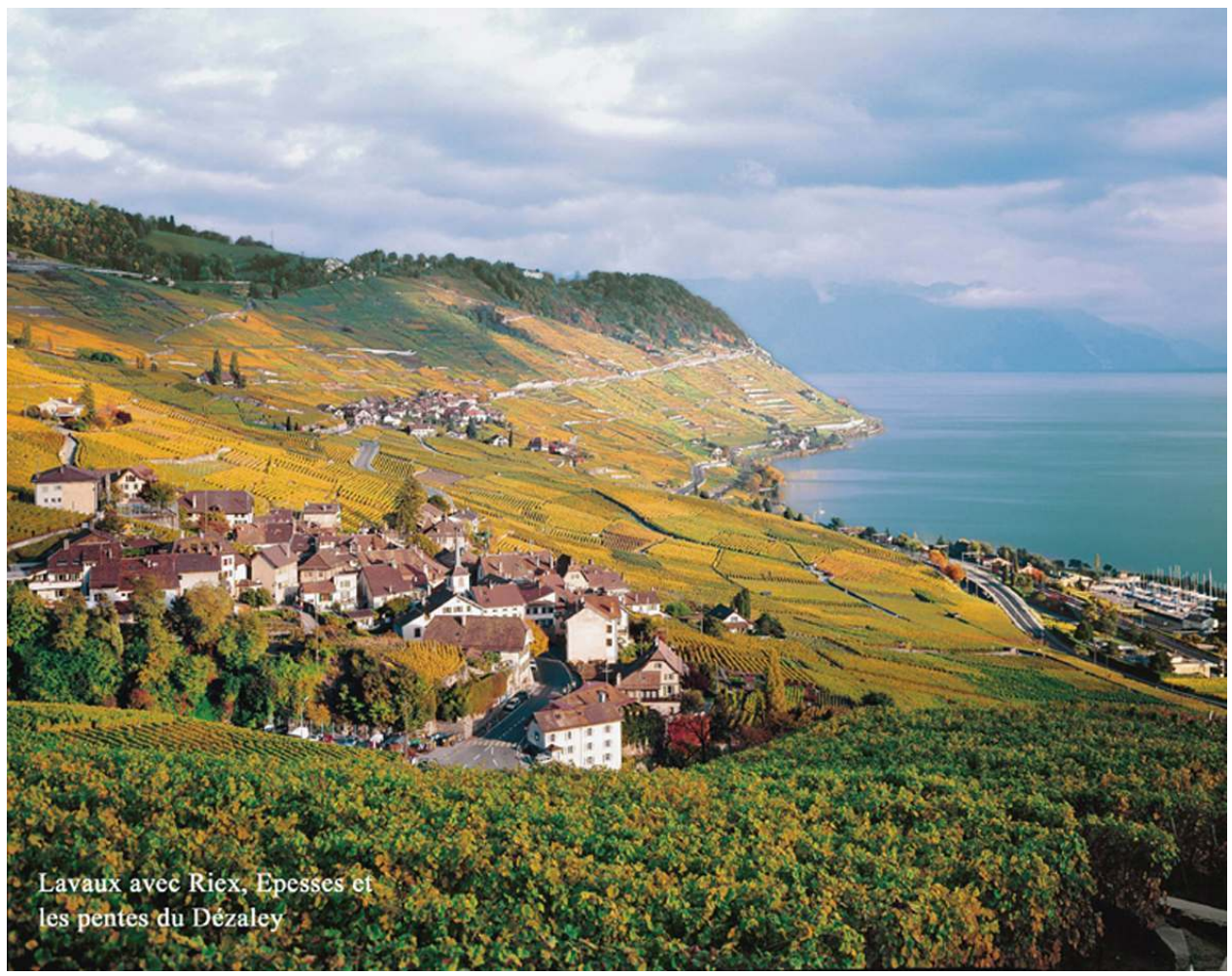

Le vignoble de Lavaux au bord du lac Léman

Phot. D. Glauser (c) D. Glauser 
Figure 3

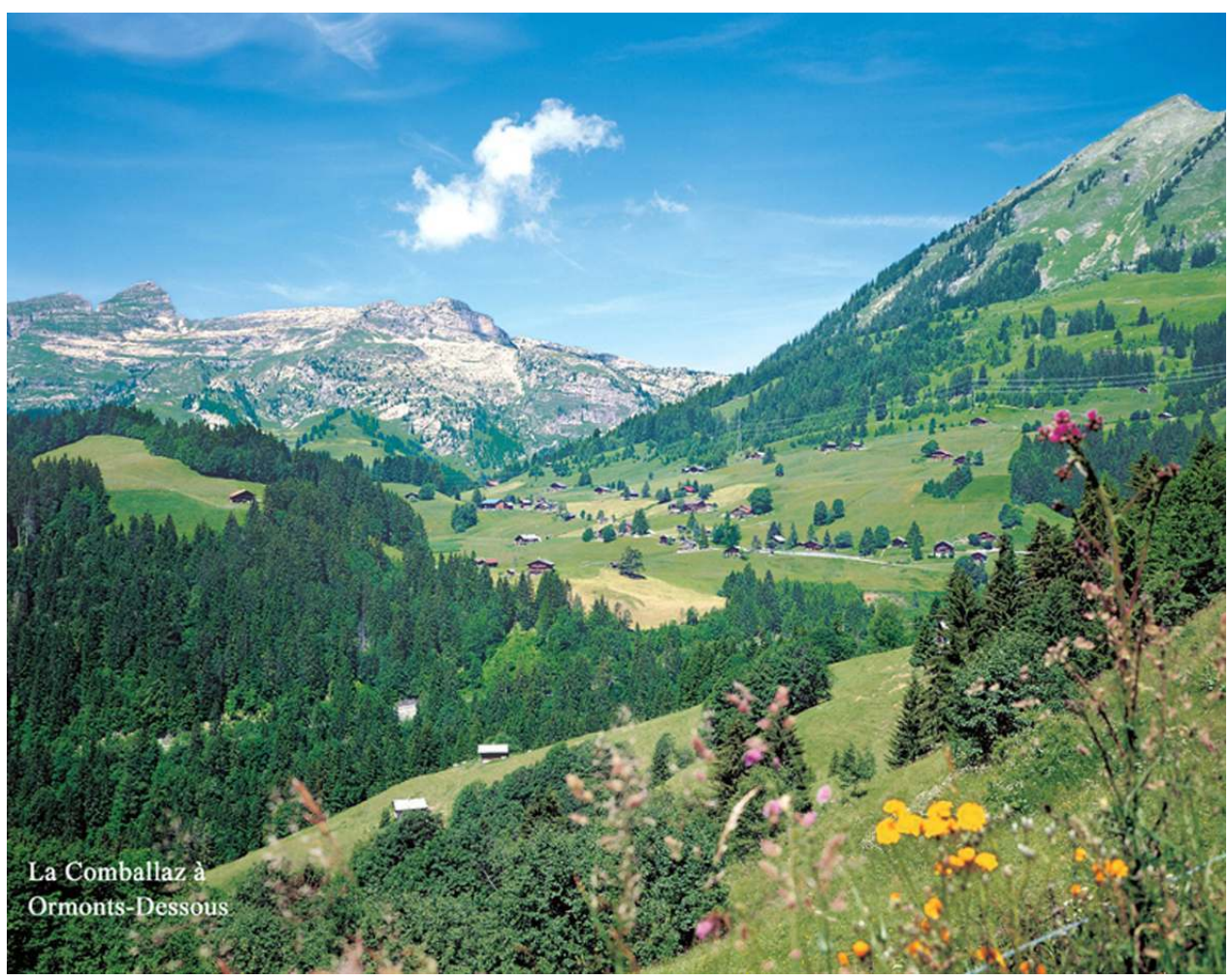

La Comballaz aux Ormonts-Dessous dans les préalpes vaudoises Phot. D. Glauser (c) D. Glauser

Figure 4

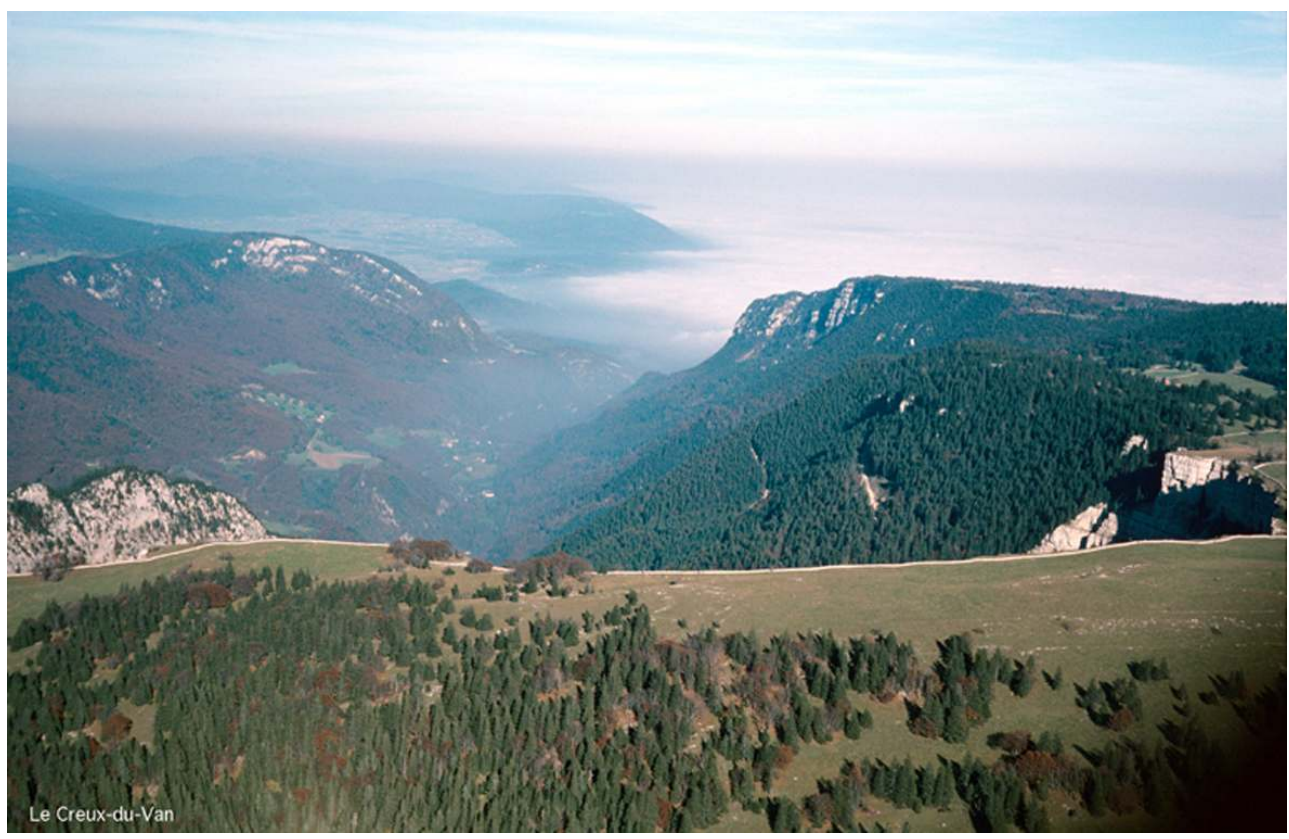

Le Creux-du-Van et les gorges de l'Areuse Phot. D. Glauser @ D. Glauser 


\section{Forme des communes}

2 Les territoires communaux sont limités entre eux par la topographie. Ils ont souvent été pris sur les forêts et les clairières de défrichement, où leurs traces restent lisibles (fig. $\mathbf{n}$ $\circ 5)$.

Figure 5

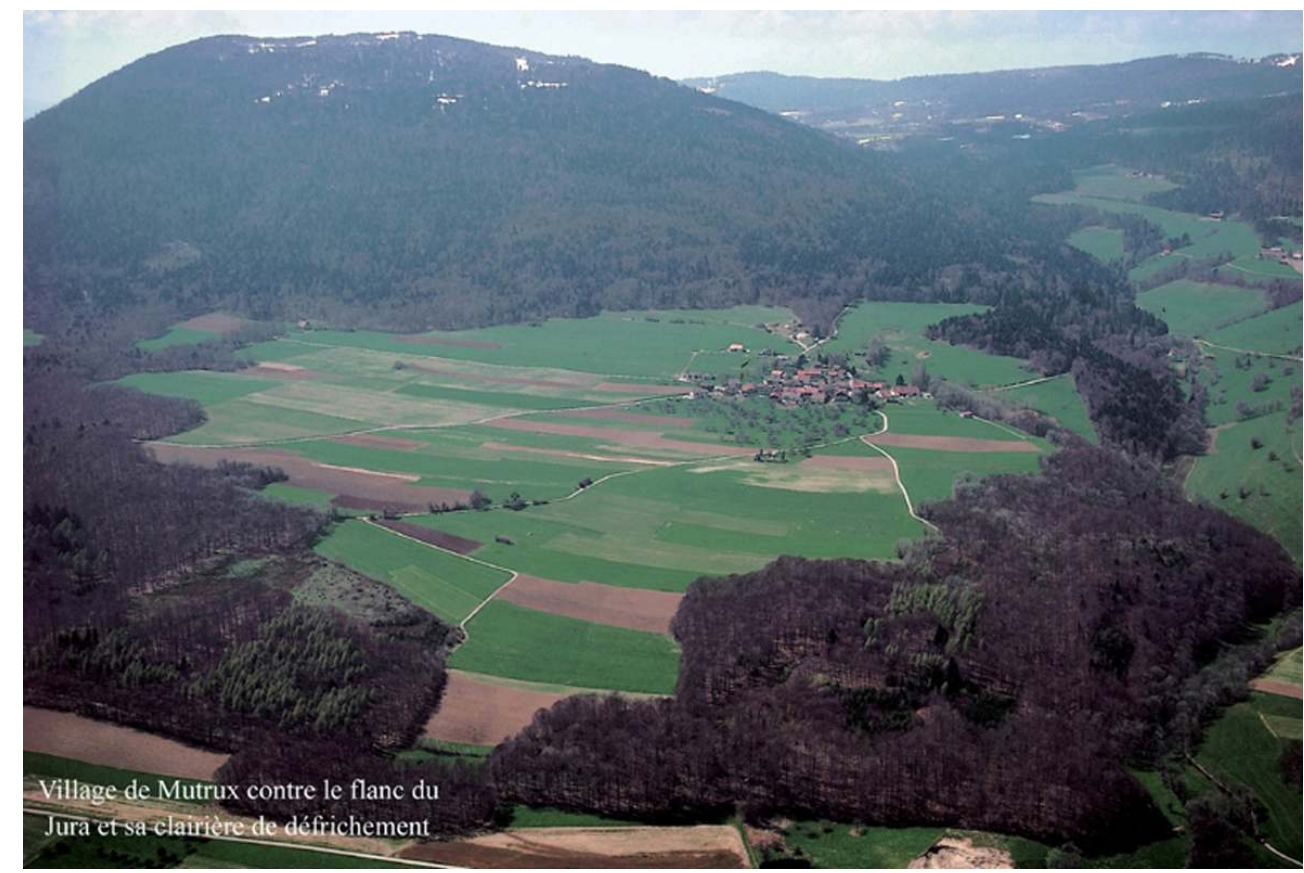

Le village de Mutrux et sa clairière de défrichement

Phot. D. Glauser @ D. Glauser

3 Les communes de montagne sont généralement de grandes dimensions (3135 ha à Provence, 6322 à Ormont-Dessous, 9905 au Chenit); elles étaient subdivisées en unités plus petites dans le passé, par exemple les «seytes» des Ormonts ou les «dizains» de Sainte-Croix à l'époque bernoise. Les villages et les hameaux qui composent la commune de L'Abbaye à la vallée de Joux jouissaient d'une certaine indépendance ; ils sont toujours propriétaires de biens fonciers. La montagne a été colonisée tardivement (vers le XIII siècle) par rapport au Moyen-Pays. L'habitat du Jura vaudois se développe d'abord de manière concentrée. Les défrichements progressent à partir du $\mathrm{XV}^{e}$ siècle, selon un schéma plus individualiste avec une dispersion des maisons en hameau, puis par unité d'exploitation. Dans les montagnes neuchâteloises, la colonisation prend son essor au XIII e siècle selon des schémas de dispersion en lanière sur les flancs des vallées ou en nid d'abeille sur les espaces plus ouverts.

4 Les communes du Moyen-Pays sont beaucoup plus petites, conditionnées par la viticulture et la culture intensive des céréales. Ces dernières ont favorisé une exploitation du finage en «champs ouverts» (openfield) conditionnée par l'assolement triennal collectif ; ce système est abandonné au début du XIX ${ }^{e}$ siècle déjà. 


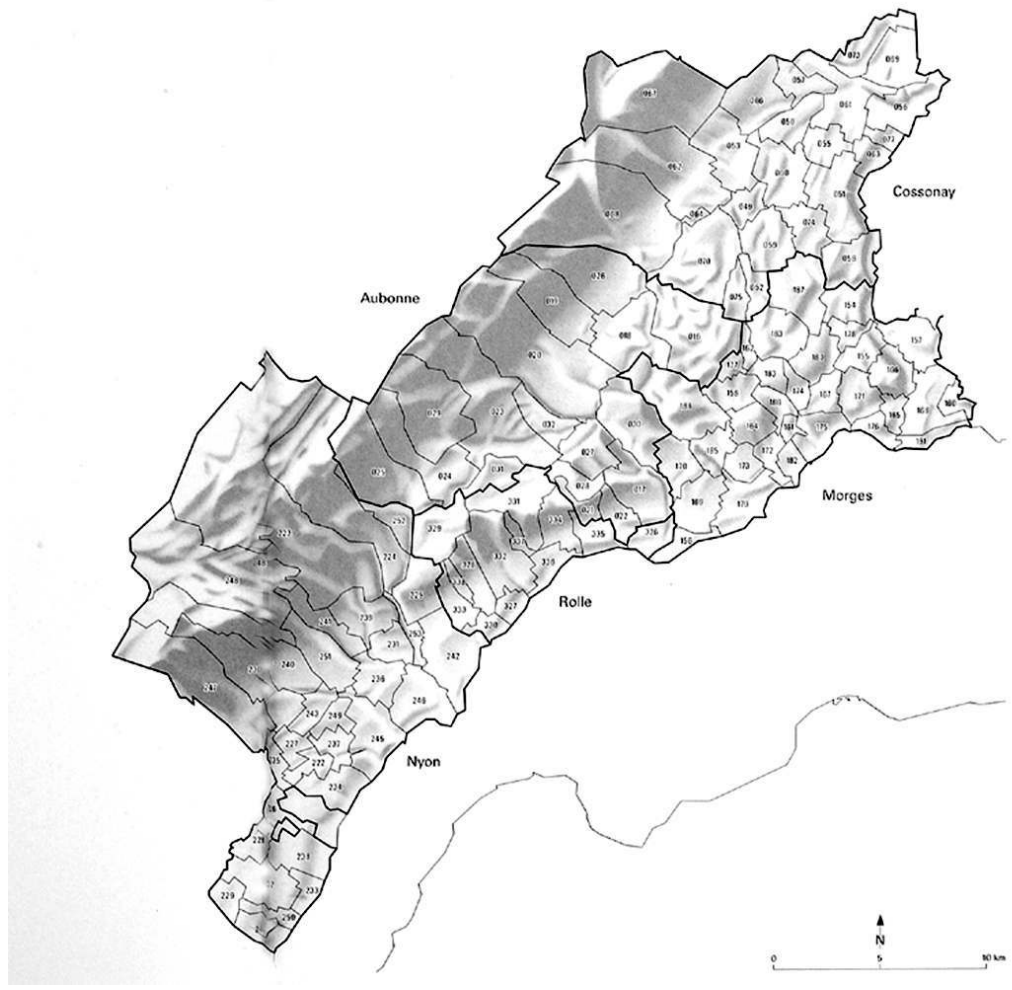

La région de La Côte sur le flanc sud-est du Jura

Phot. D. Glauser ( $)$ D. Glauser

5 La région de La Côte se subdivise en trois niveaux en fonction de l'altitude et du genre d'exploitation (fig. $\left.\mathbf{n}^{\circ} \mathbf{6}\right)$ :

- Dans la zone riveraine du Léman, la vigne paraît avoir favorisé la formation de petits territoires. L'explication réside certainement dans le fait que le rendement plus bénéfique de cette culture, pratiquée de manière intensive, et l'exportation des vins ont généré et génèrent toujours des revenus permettant d'assurer largement l'autonomie de petites entités communales. Les villages sont généralement localisés à proximité du vignoble qui nécessite des soins constants. En zone viticole, on pratique ou pratiquait toujours l'élevage, complément indispensable pour la production de fumier utilisé comme engrais.

- Au-dessus, sur un niveau médian, la surface des communes augmente (env. 500 ha). Le vignoble n'occupe plus que les coteaux les mieux exposés, alors que les cultures et l'élevage gagnent en importance.

- Le niveau supérieur comprend des vastes territoires (env. 1750 ha) qui s'étendent du pied de la montagne jusqu'à son sommet, couronné par les pâturages d'altitude; l'élevage et l'exploitation forestière constituent la principale spécialisation de cette zone. La vigne n'est plus présente, sauf sous une forme résiduelle dans le district de Nyon. Ces grandes communes allongées (voir fig. $n^{\circ} 6$ ) occupent, par bandes successives, l'ensemble du versant jurassien de la partie supérieure des districts de Nyon, d'Aubonne et de Cossonay. On retrouve cette disposition sous une forme moins claire dans la région de Grandson. D'une régularité presque sans faille, la typologie de ces territoires constitue un véritable cas d'école. Ils comprennent tous une importante part de forêts et de pâturages d'alpage qui, à elle seule, représente en moyenne les deux-tiers de la surface totale. 
L'éloignement de la chaîne jurassienne par rapport au Léman et aux Préalpes définit, sur le Moyen-Pays vaudois, un espace qui s'ouvre en direction de l'est, à partir du district de Cossonay. La région comprise entre le Gros-de-Vaud et la Broye ne se caractérise pas par un relief aussi accentué que celui décrit précédemment. On y rencontre un plateau, celui d'Echallens, et une série de vallonnements qui prennent naissance à partir du Jorat et $\mathrm{du}$ piémont des Préalpes fribourgeoises. Cette topographie relativement douce conditionne aussi l'organisation des communes selon trois grandes catégories (fig. $\mathbf{n}^{\circ} \mathbf{7}$ ).

Figure 7

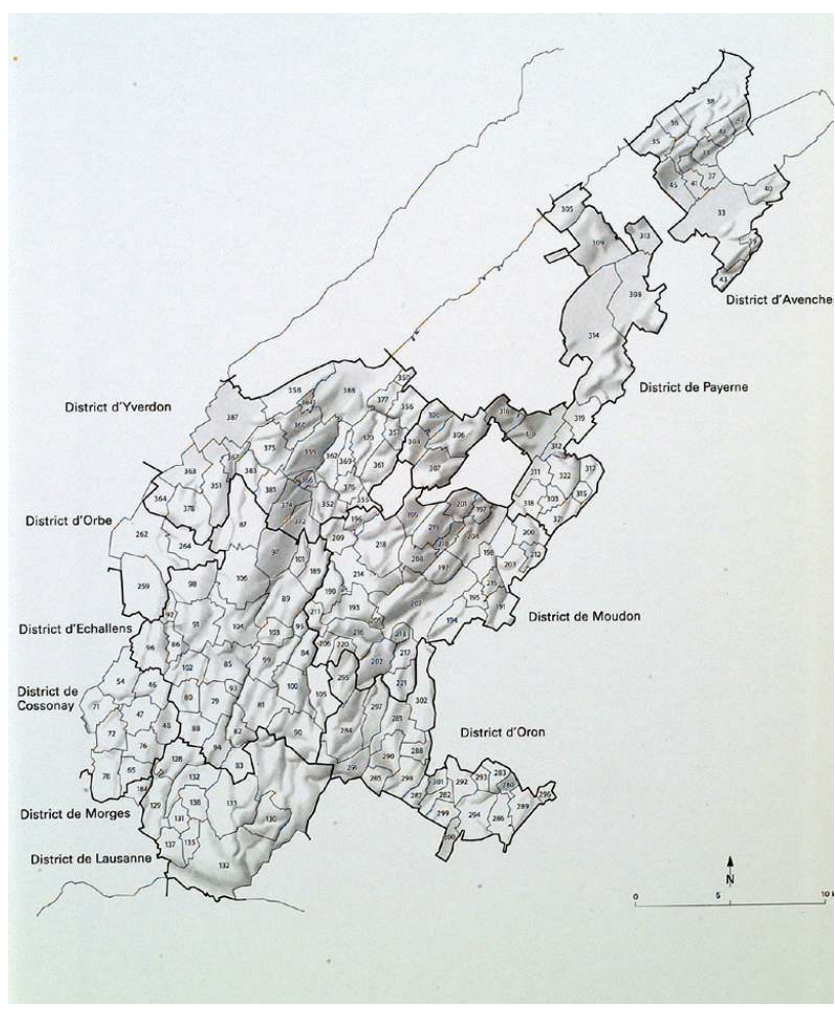

Le Gros-de-Vaud et la Broye

Phot. D. Glauser (c) D. Glauser

$7 \quad$ Les espaces ouverts du plateau d'Echallens ont généré des territoires de forme alvéolaire. Cette structure, connue également sous le nom de nid d'abeilles avec une disposition penta- ou hexagonale, se caractérise par un habitat concentré au milieu du terroir et des liaisons routières en étoile vers les entités voisines.

8 Au nord-est, dans le prolongement du plateau d'Echallens, le vallonnement se marque et s'oriente en direction du lac de Neuchâtel et de la plaine de la Broye. Le réseau hydrographique cloisonne et structure ainsi les communes qui prennent une forme allongée (voir fig. $\mathrm{n}^{\circ} 7$ ); cette dernière se répercute sur la configuration des villages, généralement dominée par une organisation linéaire avec une croisée orthogonale de chemins. 


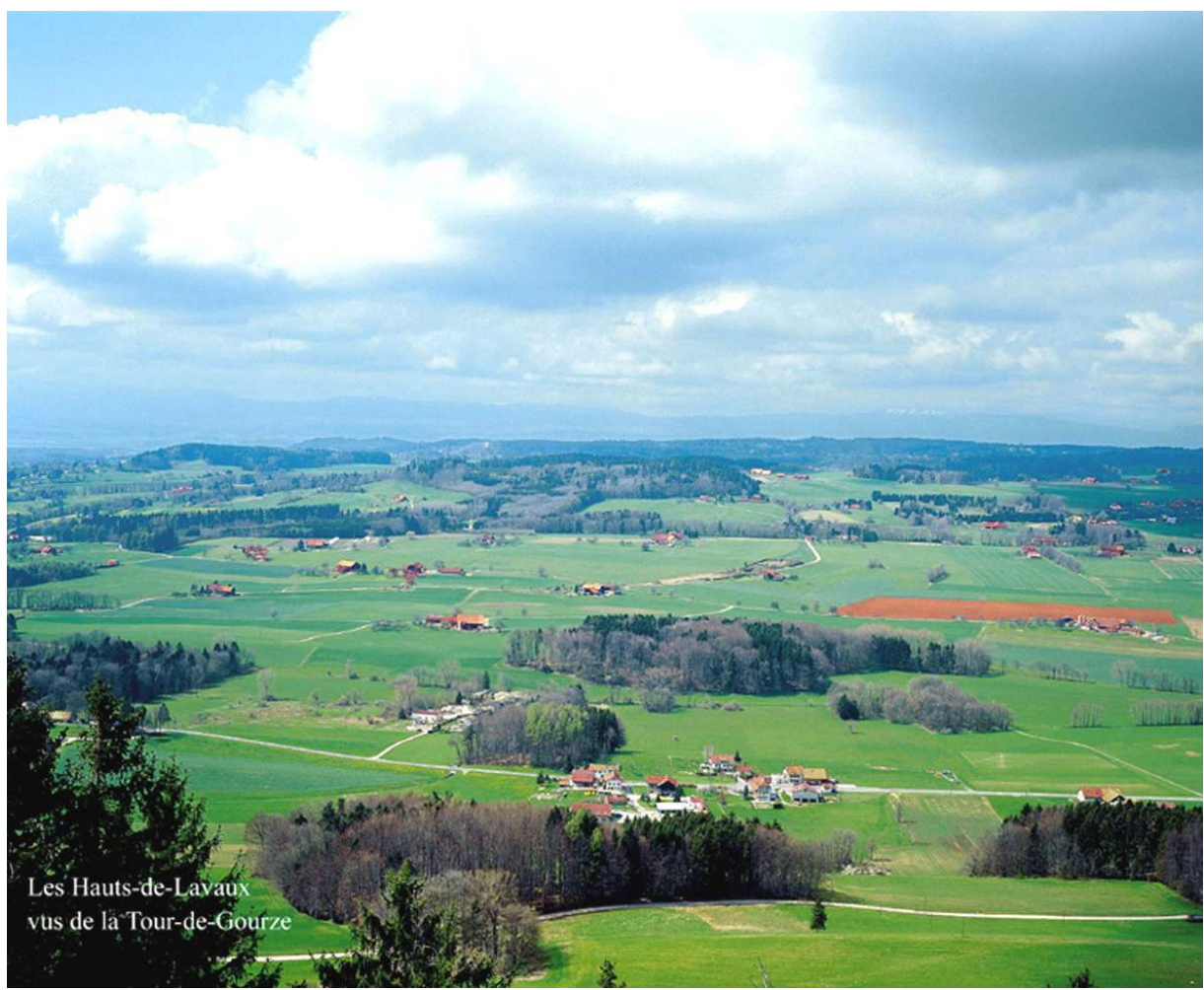

Les Hauts-de-Lavaux vus de la Tour-de-Gourze

Phot. D. Glauser (c) D. Glauser

Une vallée, celle de la Broye, conditionne toute la partie est du canton de Vaud. Selon son tracé, on distingue :

- La Haute-Broye à laquelle on peut rattacher les parties élevées du Jorat (fig. $\mathbf{n}^{\circ} \mathbf{8}$ ). Elle se caractérise par une dispersion de l'habitat sous la forme de hameaux ou par unité d'exploitation. Cette dispersion résulte à la fois d'une colonisation médiévale assez tardive et d'une spécialisation dans l'élevage. Les communes de la partie supérieure du district d'Oron sont de faibles dimensions ; elles présentent une forme allongée compartimentée par le réseau hydrographique. Dans le Jorat, les territoires sont assez vastes, comme à Savigny, au Mont ou à Lausanne, par exemple ; ils étaient en liaison étroite avec le vignoble de Lavaux dont ils formaient l'étage supérieur dévolu à l'élevage et à la production d'engrais. (fig. $\mathbf{n}^{\circ}$ 9)

- La Basse-Broye débute à Moudon et s'étend jusqu'au débouché de la vallée dans la plaine, sur la commune de Payerne (fig. $\mathbf{n}^{\circ}$ 9). Ses versants sont partiellement boisés, souvent de manière continue dans les parties où le relief est bien marqué. La forêt cloisonne les espaces agricoles, surtout au nord-ouest, contrairement à la partie qui jouxte le canton de Fribourg. En toute logique, la topographie définit également des territoires allongés où dominent les villages organisés linéairement dans l'axe de la vallée, par opposition au système alvéolaire du plateau d'Echallens.

- La plaine de la Broye, comme celle de l'Orbe, formait un obstacle à la colonisation. Les villages se concentrent à la base des versants, sur les premières collines, pour se mettre à l'abri des inondations, fréquentes jusqu'à la fin du XIXe siècle. Le Vully forme la ligne de crête qui sépare la plaine de la Broye et le lac de Morat de celui de Neuchâtel. 
Figure 9

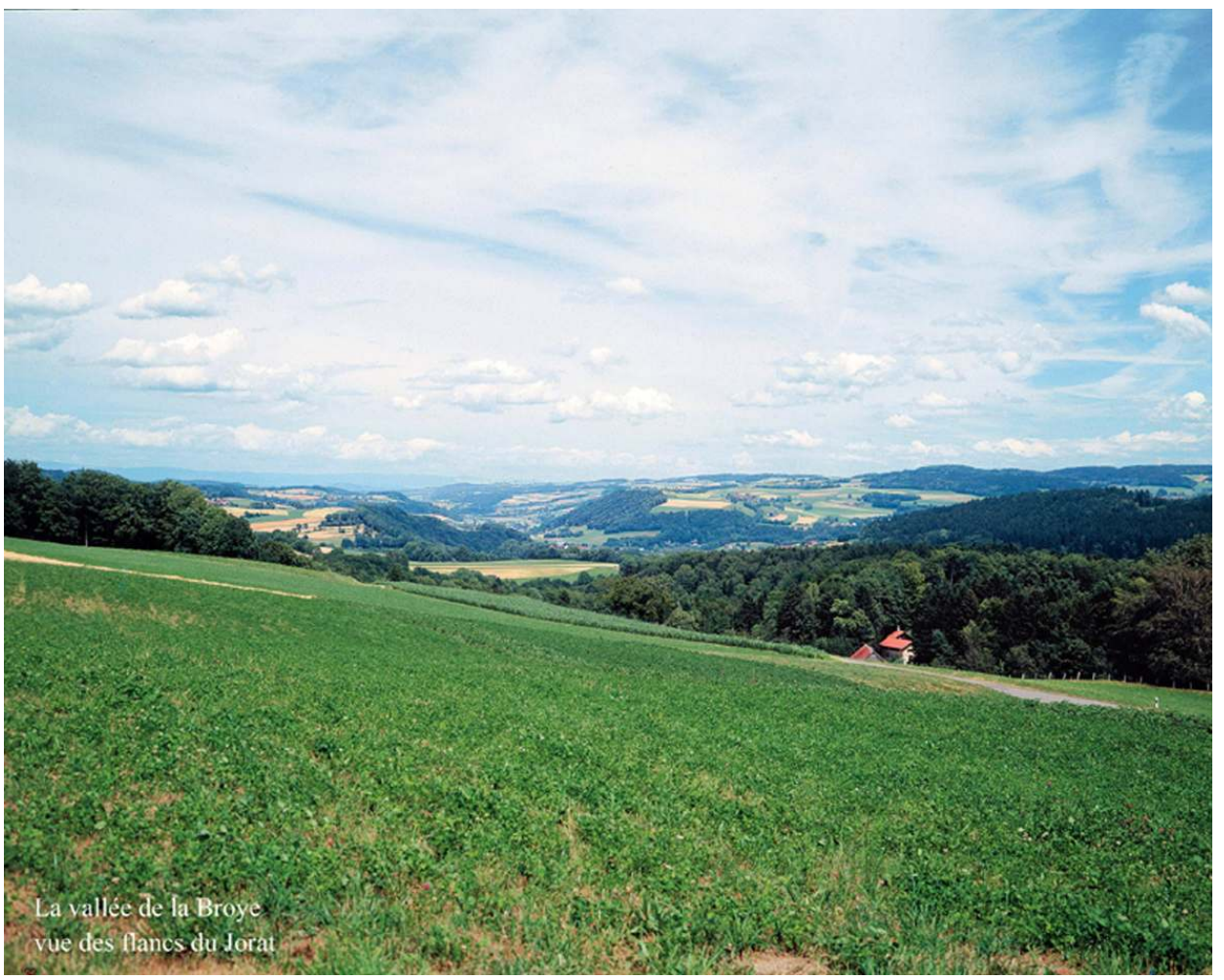

La vallée de la Broye vue des flancs du Jorat

Phot. D. Glauser (c) D. Glauser 
Figure 10

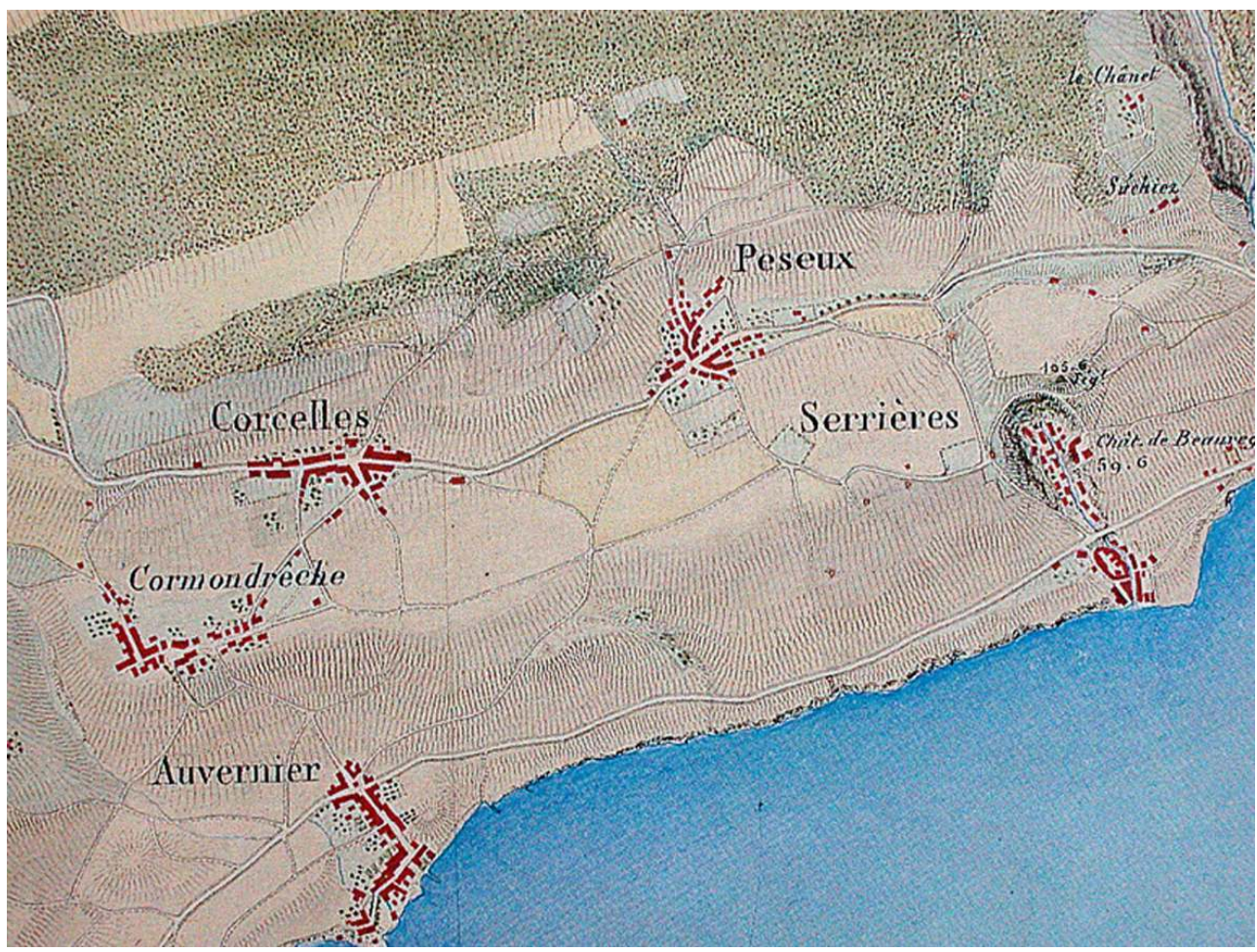

Les villages à l'ouest de Neuchâtel selon la carte Osterwald 1838-1845

Phot. D. Glauser @ D. Glauser

10 Les communes littorales du canton de Neuchâtel ont une orientation viticole bien marquée (fig. $\mathbf{n}^{\circ} \mathbf{1 0}$ ). Leurs villages présentent des organisations linéaires, soit horizontales (par exemple Corcelles) ou montantes (Cressier), soit combinées entre elles (Auvernier). La pression immobilière est particulièrement forte dans ces régions et a entraîné à partir de la fin du XIX ${ }^{\mathrm{e}}$ siècle des développements qui ont gagné sans cesse en importance. L'exemple de Peseux est particulièrement parlant avec une disparition presque complète des vignes.

11 Territoire intermédiaire entre lac et montagne, le Val-de-Ruz forme une sorte de «barque » dont les côtés sont limités par le synclinal de Chaumont/Chasseral et celui qui va du Mont-d'Amin au Mont-Racine, en passant par la Vue-des-Alpes et Tête-de-Ran (fig. $\mathbf{n}^{\circ}$ 11). Son versant nord-ouest est occupé par des communes qui incluent jusqu'à trois vallons successifs au-delà du Mont-d'Amin. Les villages situés dans les vallées, Fontaines, Engollon, Boudevilliers et Coffrane, sont plus ou moins centrés sur leur terroir (espace dévolu aux cultures). A l'exception d'Engollon, ils comptent tous aussi une partie de montagne. Fontaines se divise en deux parties distinctes; la part de montagne y est importante et se répartit de part et d'autre du col de la Vue-des-Alpes. 


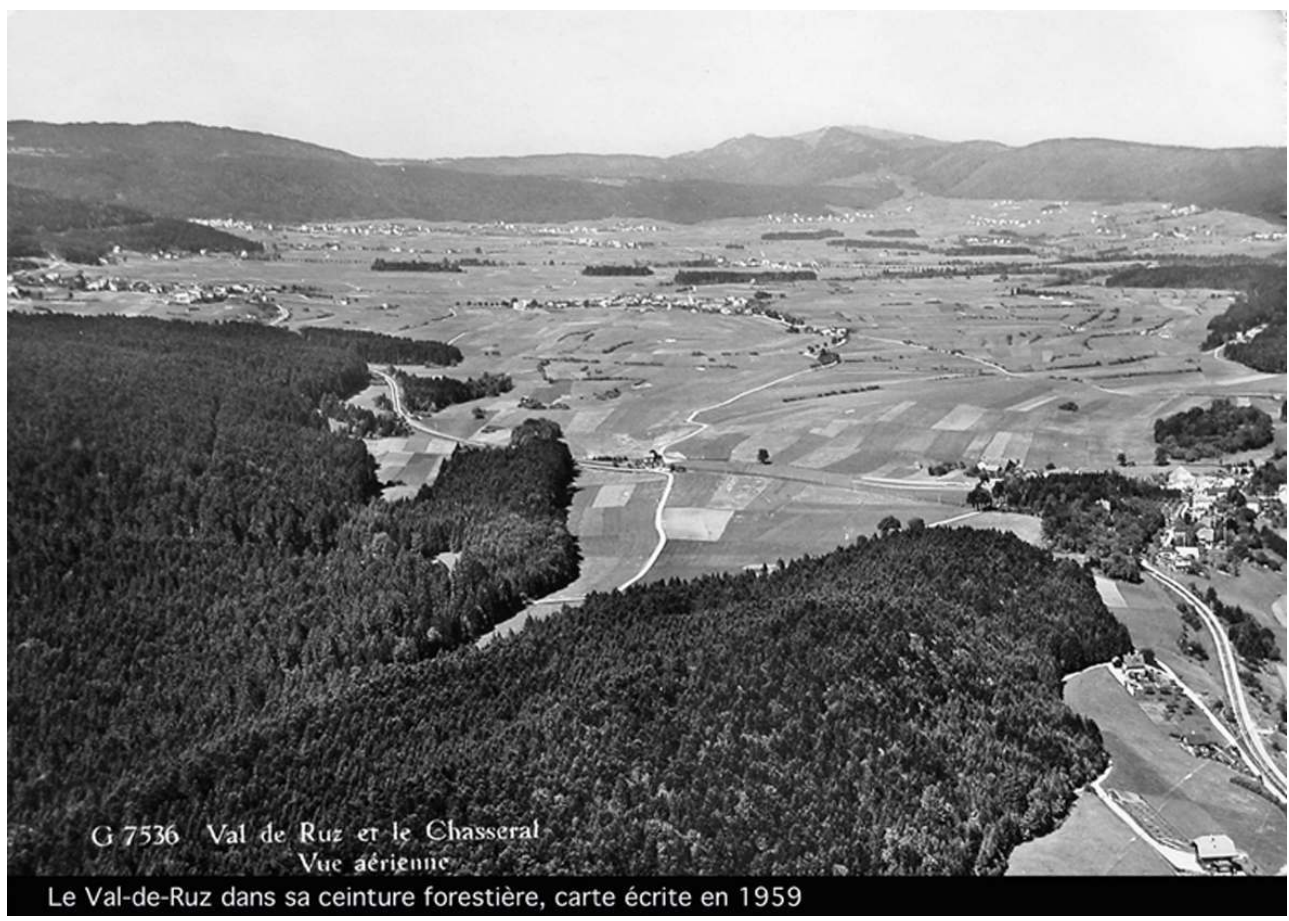

Le Val-de-Ruz au milieu du XXe siècle

Phot. D. Glauser (c) D. Glauser

12 La colonisation du fond de la vallée est ancienne, comme l'attestent des vestiges préromains (tumuli) et gallo-romains (villas). Certains toponymes indiquent eux aussi une origine gallo-romaine des villages comportant des terminaisons caractéristiques en -ier (Cernier, Savagnier). Vilars, Villiers, Boudevilliers et Malvilliers contiennent un suffixe dérivant du bas-latin «villa » signifiant «qui appartient au domaine de ». L'habitat de cette région se concentre dans des villages ou des hameaux. En revanche, les vallons de la partie supérieure du Val-de-Ruz ont été colonisés plus tardivement; l'article précède le nom de lieu selon un usage qui n'intervient généralement qu'à partir du XII e siècle (La Joux-du-Plâne, Le Côty,...). L'habitat s'y présente principalement sous la forme d'unités d'exploitation dispersées, constituées chacune d'une ferme sur son domaine (fig. $\mathbf{n}^{\circ} \mathbf{1 2}$ ). 


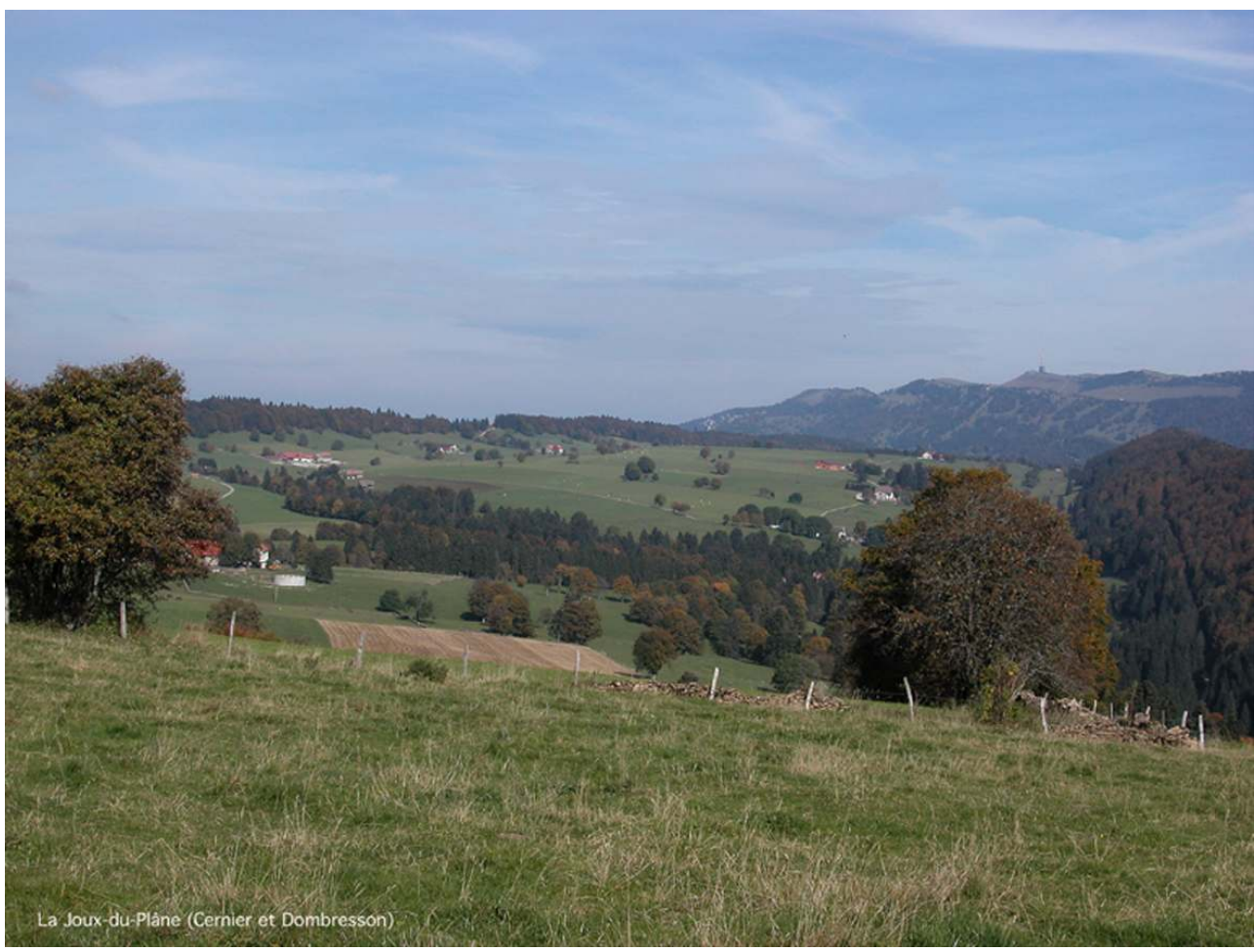

Habitat dispersé à la Joux-du-Plâne au-dessus du Val-de-Ruz

Phot. D. Glauser (c) D. Glauser

\section{Structures des villages}

La diversité des structures des villages constitue une véritable richesse patrimoniale. Les documents cadastraux élaborés aux $\mathrm{XVIII}^{\mathrm{e}}$ et $\mathrm{XIX}^{\mathrm{e}}$ siècles facilitent le travail de recherche. Leur consultation permet de compléter la lecture des cartes actuelles; ils éclairent la définition des types de base en montrant par exemple des développements secondaires qui sont encore à l'état d'ébauche.

A l'exclusion des villes neuves médiévales qui se développent après les défrichements, les petites villes doivent constituer le moteur d'où se déploie la colonisation et sur lesquelles on peut se replier en cas de crise. Hors des centres, on commence par implanter une sorte de « semis » de constructions le long du réseau routier existant, qui s'étoffera au fur et à mesure de la densification. A l'origine d'un village, on peut trouver un ordre religieux, représenté par un prieuré rural, ou un château seigneurial. En cas de voisinage, ces deux édifices sont à même de créer des pôles d'attraction qui vont conditionner l'implantation des maisons. Il est possible d'imaginer, dans le cadre d'une famille, un regroupement d'abord dans une première maison qui sera agrandie progressivement au gré des descendances successives, pour former une propriété en ordre contigu. Les plans cadastraux des $\mathrm{XVIII}^{\mathrm{e}}$ et $\mathrm{XIX}^{\mathrm{e}}$ siècles semblent confirmer cette hypothèse et révèlent toujours une forte présence des groupes familiaux dans de nombreux villages.

La terminologie existante permettant de distinguer les diverses formes d'organisation de l'habitat restait lacunaire; les recherches entreprises nous ont permis de la préciser et de 
l'enrichir. Il convient d'être attentif au fait qu'une commune peut comprendre plusieurs éléments typologiques avec une structure affirmée, un ou plusieurs hameaux et de l'habitat dispersé, conférant une certaine complexité à l'ensemble.

On distingue trois schémas principaux d'organisation (fig. $\mathbf{n}^{\circ} 13$ ) :

Figure 13

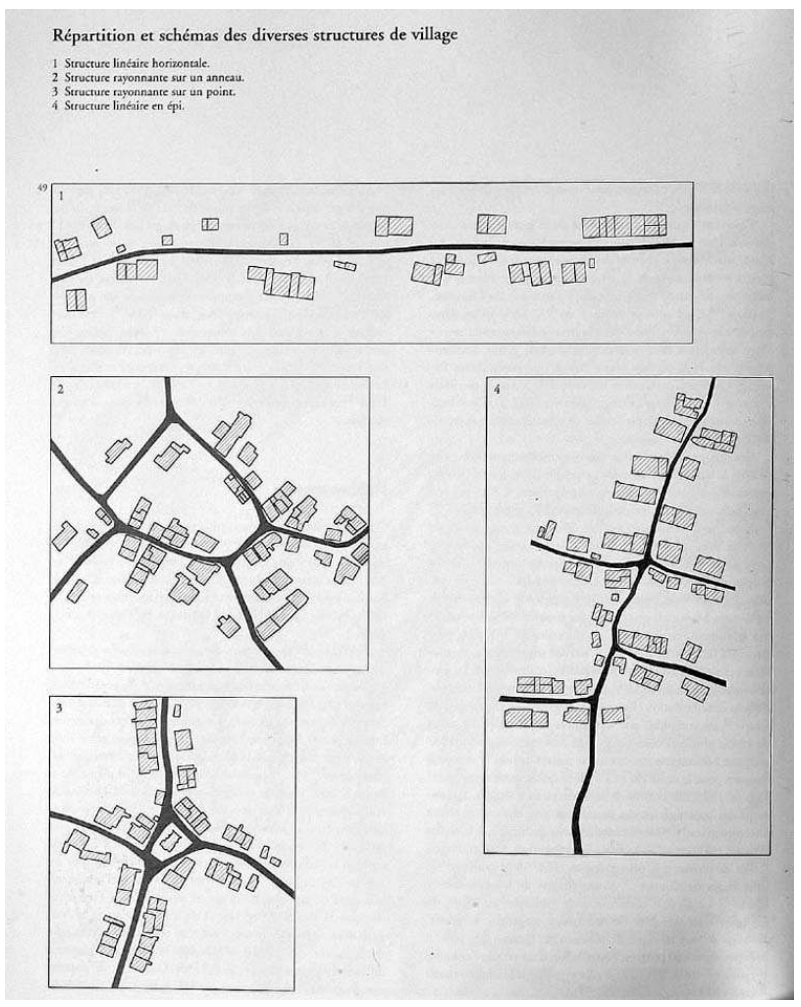

Schémas de structure des villages. 1. Structure linéaire horizontale. 2. Structure rayonnante sur un anneau. 3. Structure rayonnante sur un point. 4. Structure linéaire en épi

Phot. D. Glauser @ D. Glauser 
Figure 14

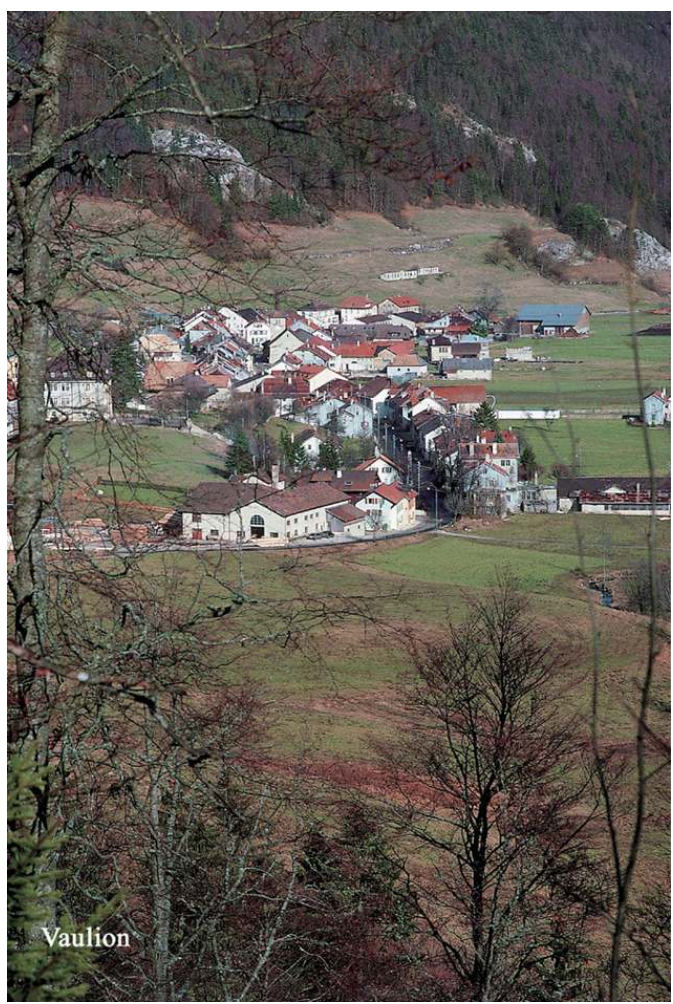

Le village-rue de Vaulion

Phot. D. Glauser @ D. Glauser

Les structures linéaires, développées en bordure d'une rue horizontale ou montante, se rencontrent surtout contre le versant du Jura ou ceux des vallées et des vallonnements qui axent les déplacements. Elles forment le schéma le plus répandu; on les subdivise en plusieurs sous-groupes : (fig. $\left.\mathbf{n}^{\circ} \mathbf{1 4}\right)$

- Les structures en épi se développent en bordure d'une rue montante; les bâtiments s'articulent perpendiculairement à celle-ci avec des faîtes de toiture qui sont parallèles aux courbes de niveaux du terrain (fig. $\mathbf{n}^{\circ}{ }^{15}$ ) (fig. $\mathbf{n}^{\circ}$ 16). Bien représenté sur le piémont jurassien, ce genre d'organisation privilégie généralement l'axe vertical de circulation, propre aux déplacements à l'intérieur de la commune. Le village est localisé à proximité des prés et des champs, dans la partie inférieure du territoire; il se développe en bordure de la route qui conduit à la montagne, sur laquelle ont été défrichés les vastes pâturages d'alpage. Provence s'organise sur la base d'un axe montant ; l'évitement routier a d'abord traversé le bâti horizontalement pour le contourner entièrement aujourd'hui (fig. $\mathbf{n}^{\circ} \mathbf{1 7}^{\text {17). }}$ 
Figure 15

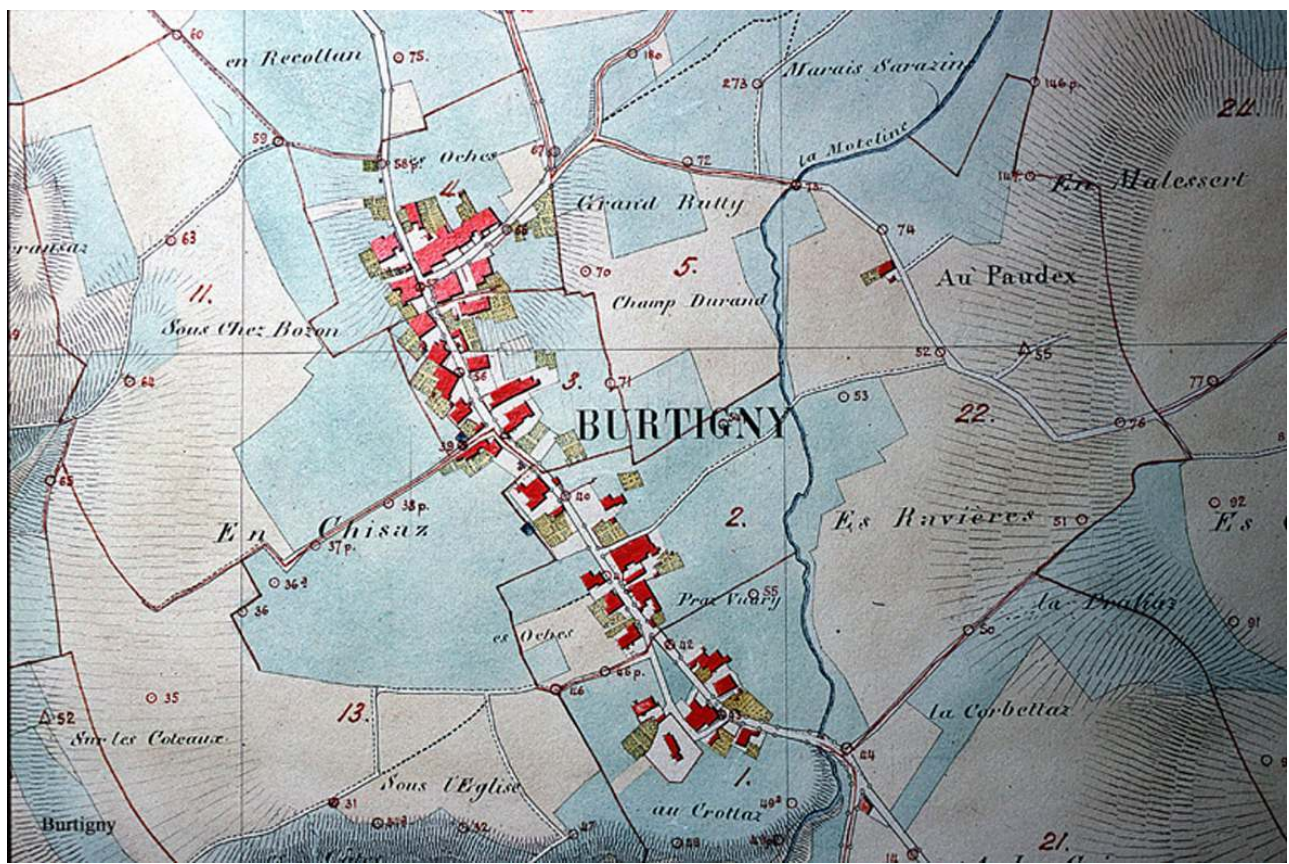

Burtigny établi en bordure d'une rue montante selon une carte de la fin du XIXe siècle conservée auX Archives cantonales vaudoises

Phot. D. Glauser (c) D. Glauser

\section{Figure 16}

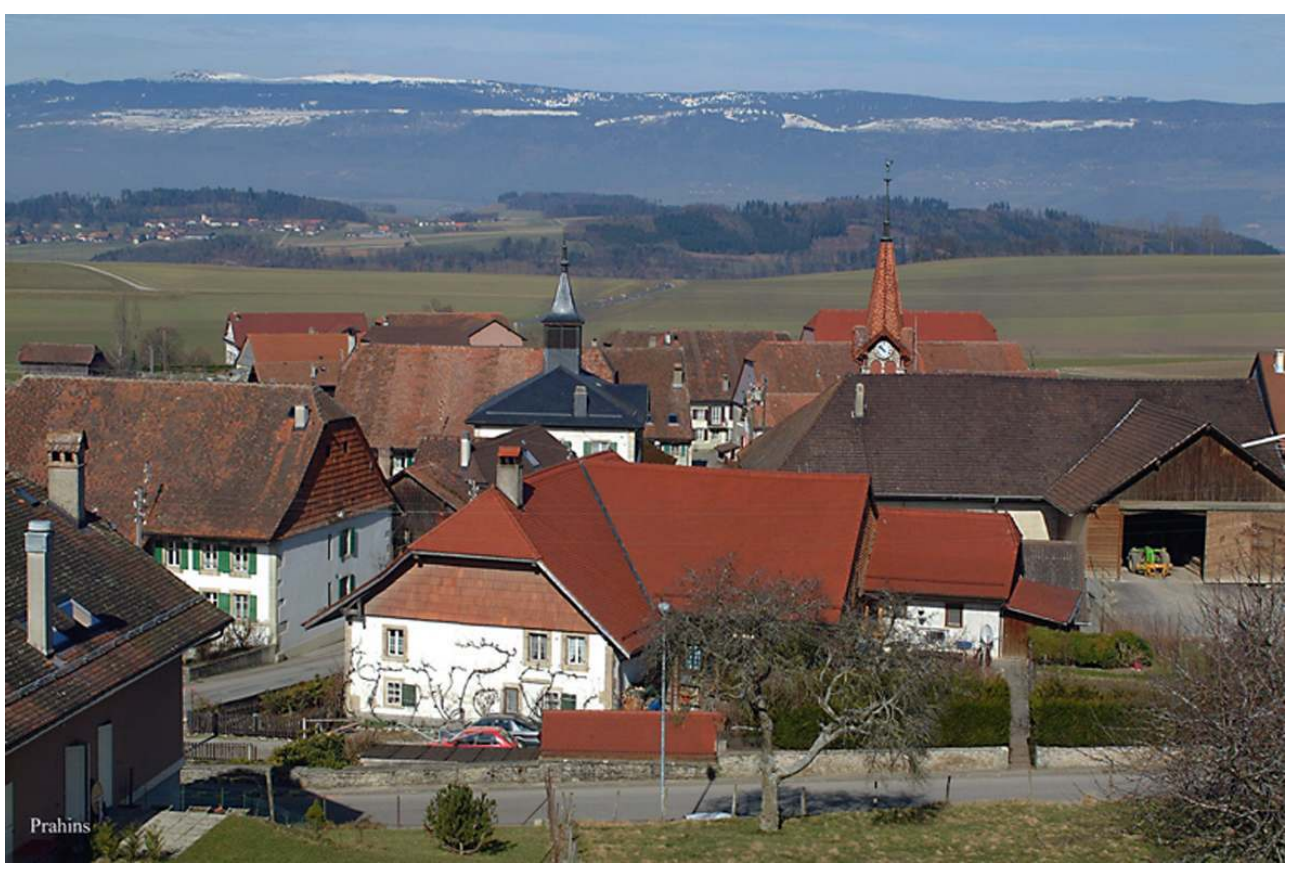

Prahins avec les faîtes de ses toits orthogonaux par rapport à l'axe de la rue Phot. D. Glauser @ D. Glauser 
Figure 17

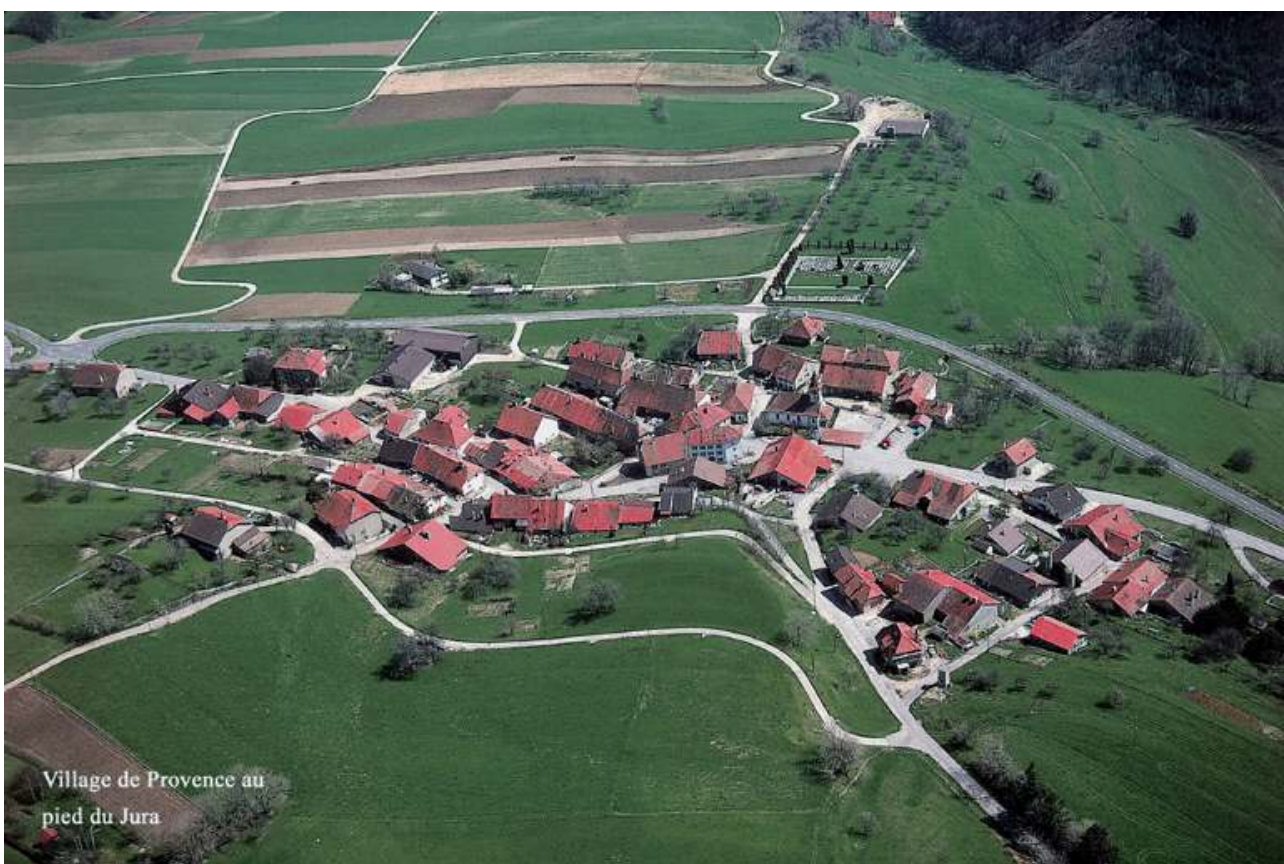

Provence et sa structure dominante en épi en bordure de la rue montant depuis le cimetière Phot. D. Glauser @ D. Glauser

Ces structures montantes connaissent deux variantes :

Figure 18

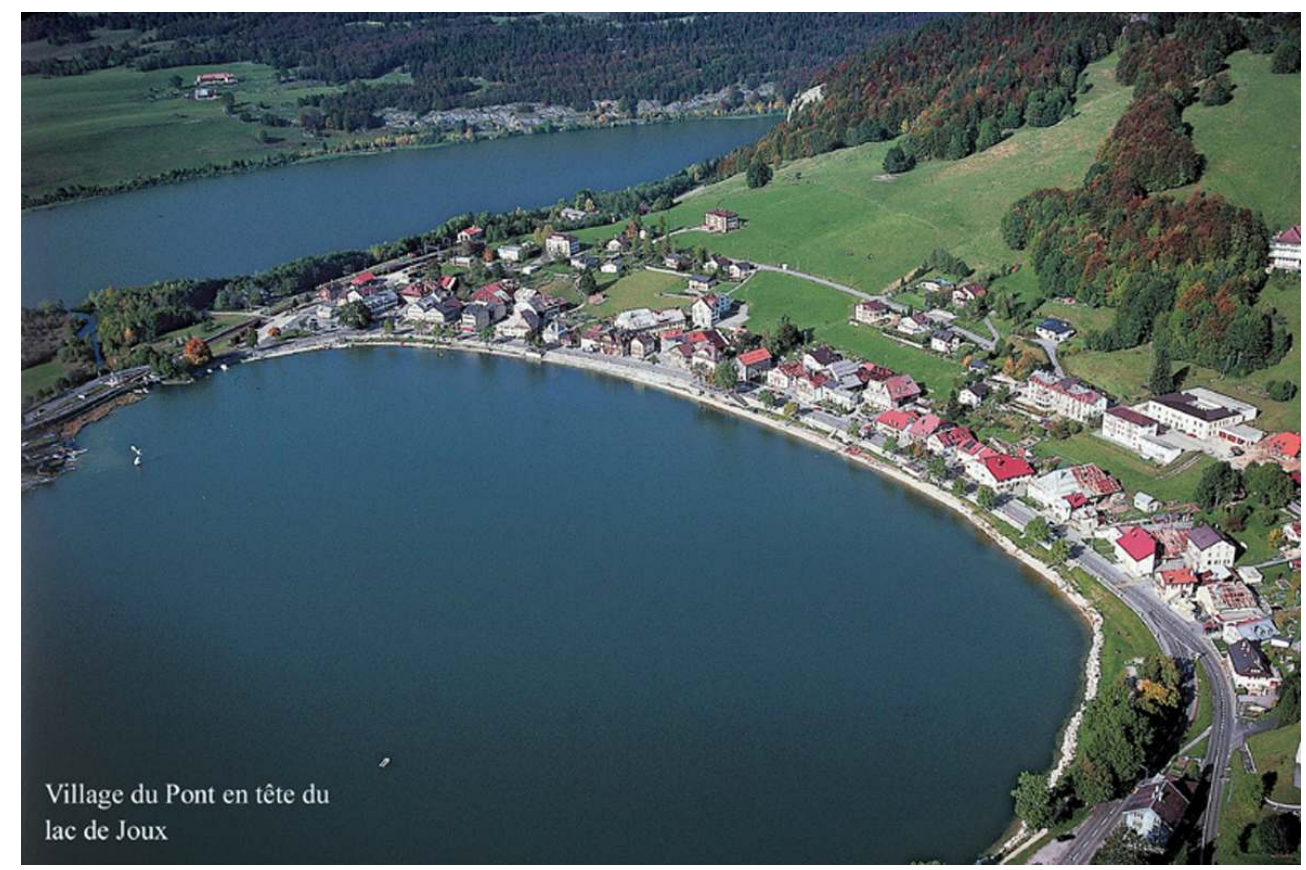

La structure en peigne du village du Pont

Phot. D. Glauser @ D. Glauser 
Les structures rayonnantes, appelées aussi étoilées, se trouvent surtout dans la zone ouverte définie par le plateau d'Echallens et son prolongement dans le district de Cossonay. Elles correspondent à une distribution alvéolaire des communes et définissent ainsi un réseau de communication rayonnant à partir du village. Les constructions se répartissent en bordure des branches formées par les routes. Il ne s'agit pas d'une règle, étant donné que les compositions linéaires peuvent aussi se combiner à des réseaux étoilés, comme évoqué précédemment. Généralement centré sur son terroir, le village

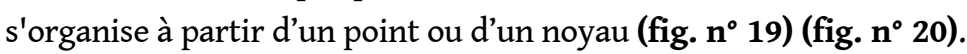

Figure 19

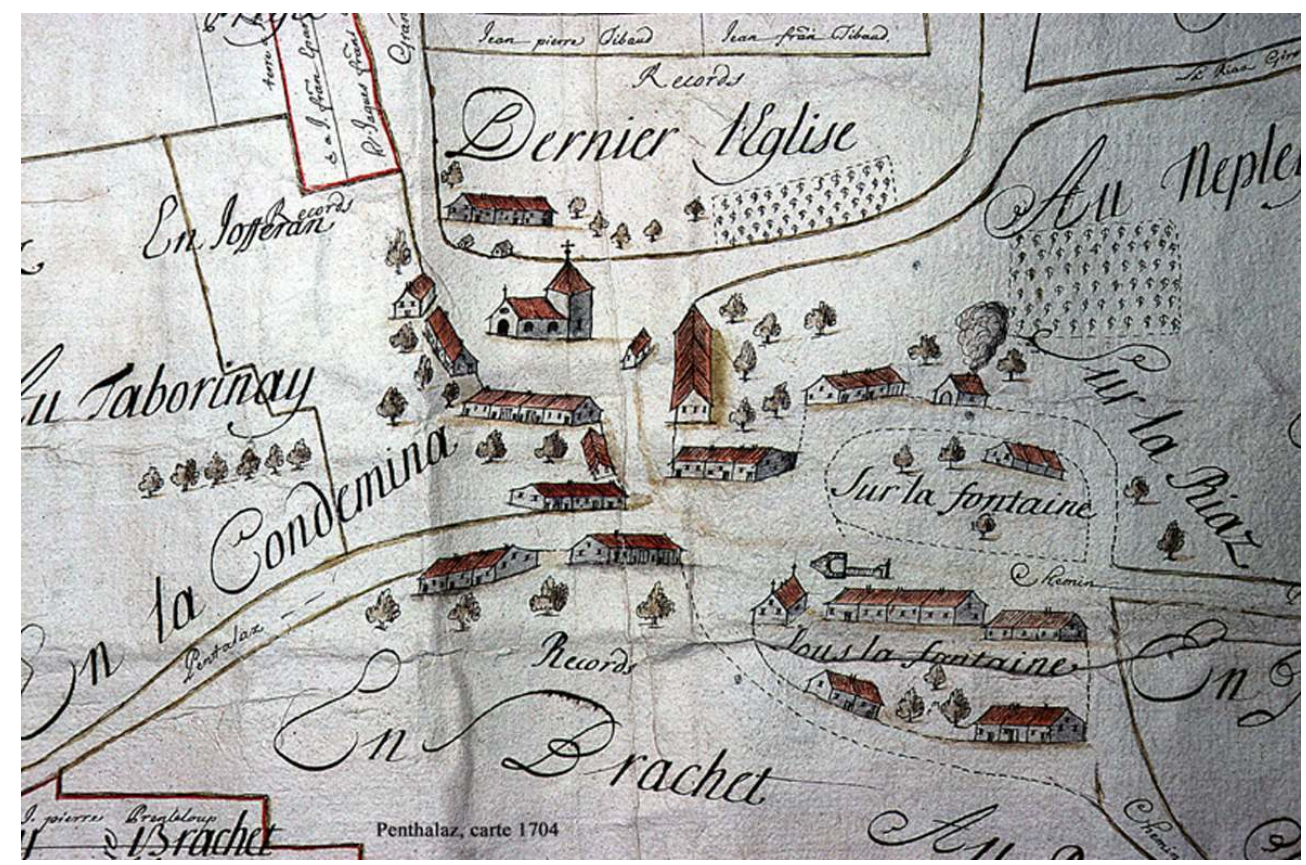

Penthalaz selon une carte de 1704 conservée aux Archives cantonales vaudoises, le réseau routier converge sur deux points

Phot. D. Glauser @ D. Glauser 


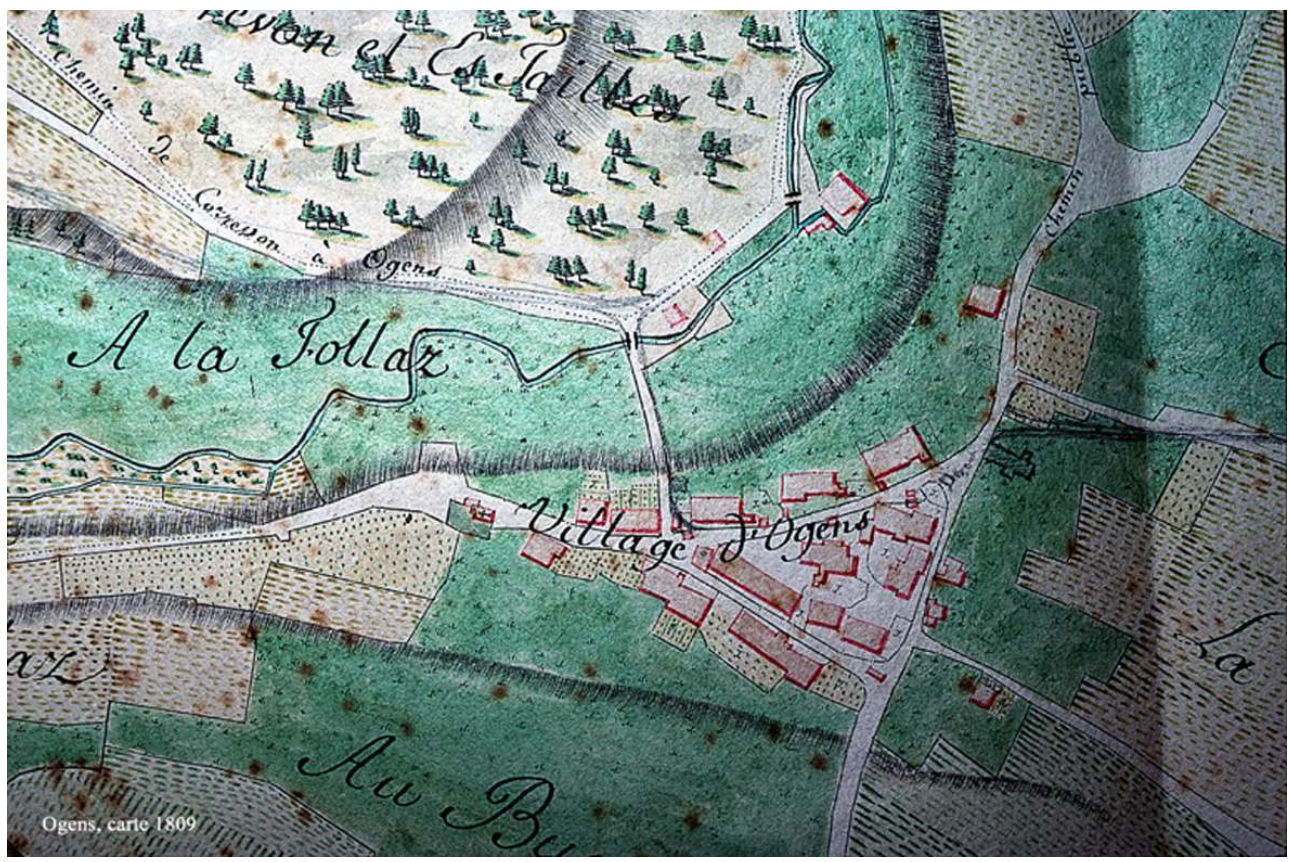

Ogens selon la carte de 1809 conservée aux Archives cantonales vaudoises, le réseau routier converge sur les angles d'un triangle

Phot. D. Glauser @ D. Glauser

A côté de ces schémas d'organisation bien typés, on trouve des formes non structurées ou restant toujours à l'état d'ébauche.

village aggloméré (ou agglomérat) correspond à la définition du « village en tas ». ${ }^{2} \mathrm{Il}$ n'est pas classable dans les autres catégories; les bâtiments sont regroupés sans ordre précis et sans qu'une structure particulière, même à l'état d'ébauche, ne soit vraiment mise en évidence. La substance routière reste faible ou extérieure et le nombre de constructions n'entre en principe pas dans sa définition. Toutefois, il correspond de manière générale à un territoire communal de petite taille; il peut aussi former un habitat localisé sur l'espace propre d'un grand territoire (Aclens près de Cossonay), ou constituer un élément proche d'une entité structurée (Le Petit-Faubourg à Montricher, Chabiez à L'Isle). On rencontre des villages agglomérés dans l'ensemble du Moyen-Pays vaudois, mais avec toutefois des dominantes dans les districts de Nyon et de Morges ainsi que dans les régions du Jorat et du Vully.

Le hameau se compose d'un nombre réduit de bâtiments, compris généralement entre cinq et quinze, en faisant abstraction des constructions modernes (villas, dépendances). Il présente souvent l'ébauche d'une structure linéaire qui rend délicate la fixation d'une norme rigide avec des groupements plus étendus. A la vallée de Joux, la combe de Derrière-la-Côte, sur la commune du Chenit, présente un bel exemple de dispersion en hameau (fig. $\mathbf{n}^{\circ} \mathbf{2 1}$ ). 


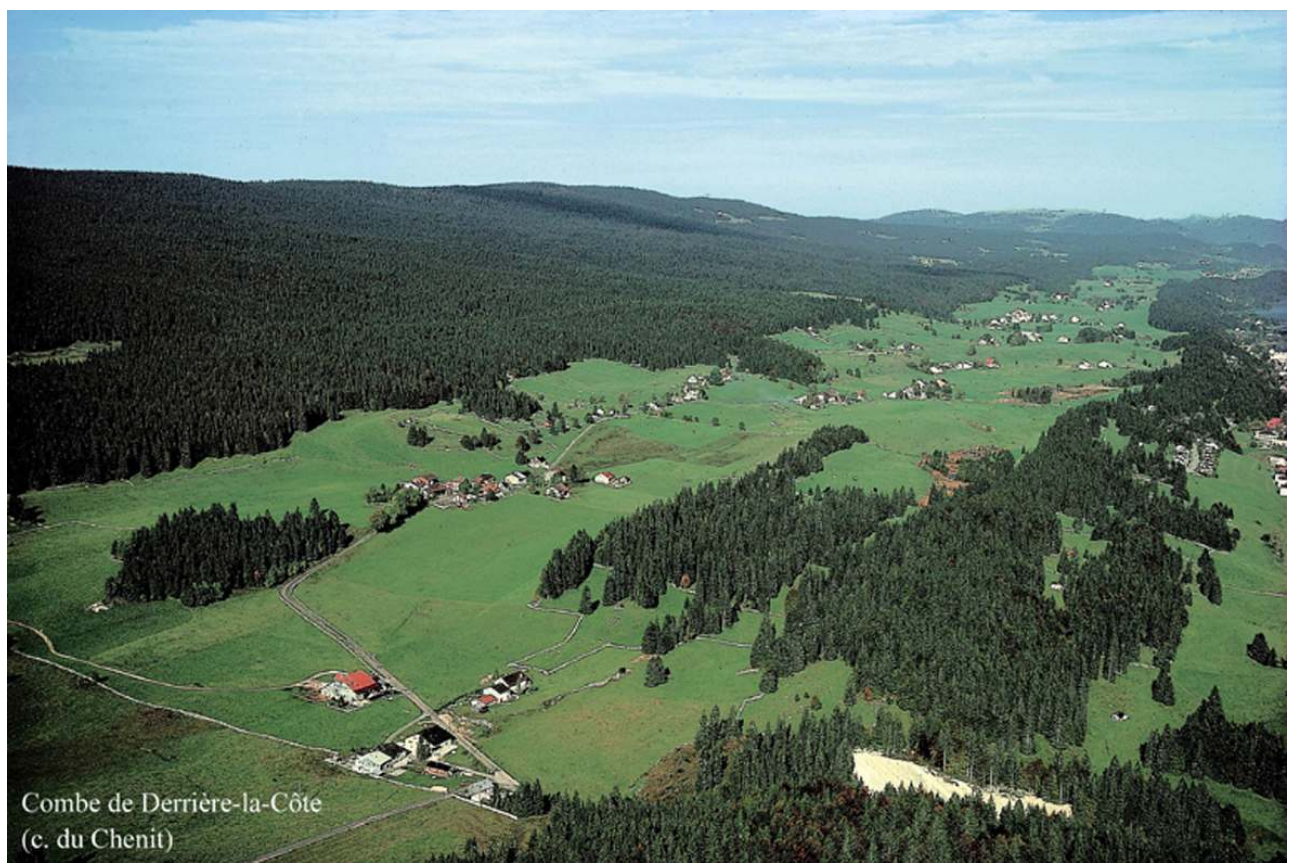

La combe de Derrière-la-Côte au nord-ouest du village du Chenit

Phot. D. Glauser @ D. Glauser

L'habitat dispersé du canton de Vaud concerne essentiellement les parties élevées du territoire (Préalpes, Jura, Jorat et Haute-Broye). Dans cette catégorie, nous n'avons pas tenu compte des maisons paysannes disséminées sur les espaces de culture après l'abolition définitive de l'assolement triennal communautaire au début du XIX ${ }^{\mathrm{e}}$ siècle. Il s'agit d'un phénomène à la fois limité et tardif. On distingue deux types de dispersion. La première par petits groupes de fermes. Ce phénomène prend naissance à partir des XII ${ }^{\mathrm{e}}$ et $\mathrm{XIII}^{\mathrm{e}}$ siècles, comme le laisse entendre la toponymie, qui révèle de nombreux noms de lieux pourvus de l'article. Le cas le plus courant se traduit par une dissémination le long d'une route ou d'un chemin, souvent à côté d'une organisation plus marquée. La seconde comprend une dispersion par unité d'exploitation. Bien visible à Epalinges sur la carte de 1836, à côté de quelques petits groupements et du hameau qui constituait alors le village, sa lecture a été profondément modifiée par une urbanisation récente qui touche l'ensemble du territoire (fig. $\mathbf{n}^{\circ} \mathbf{2 2}$ ). Le même phénomène se retrouve sur les communes proches du Mont et sur les hauts de celle de Lausanne. 
Figure 22

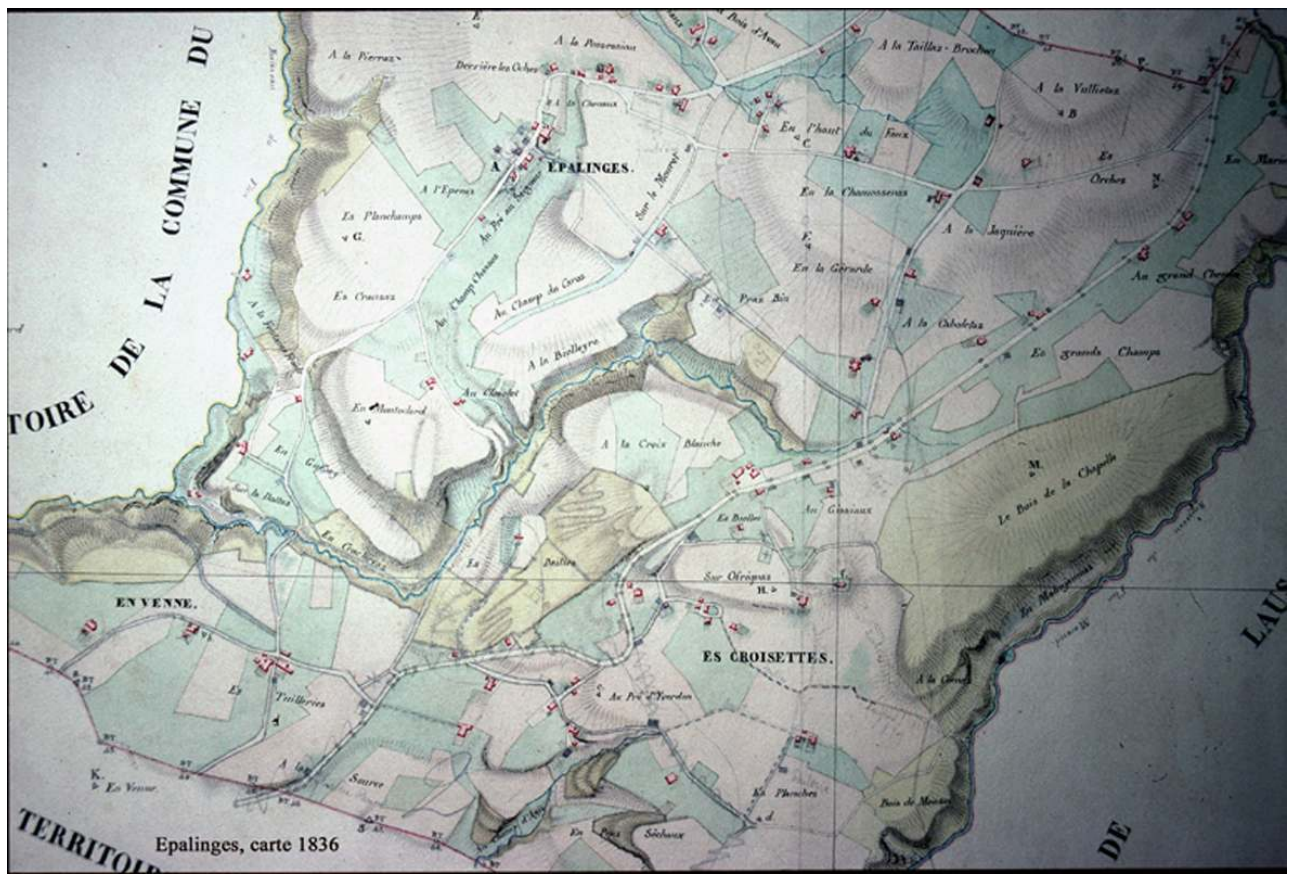

L'habitat dispersé d'Epalinges selon la carte de 1836 conservée aux Archives cantonales vaudoises Phot. D. Glauser (c) D. Glauser

Dans les montagnes neuchâteloises, les villages organisés sur la base d'un schéma concentré sont exceptionnels (Les Ponts-de-Martel, La Sagne, La Brévine). L'habitat permanent s'y répartit de manière dispersée selon trois schémas : (fig. $\left.\mathbf{n}^{\circ} \mathbf{2 3}\right)$ 


\section{Figure 23}

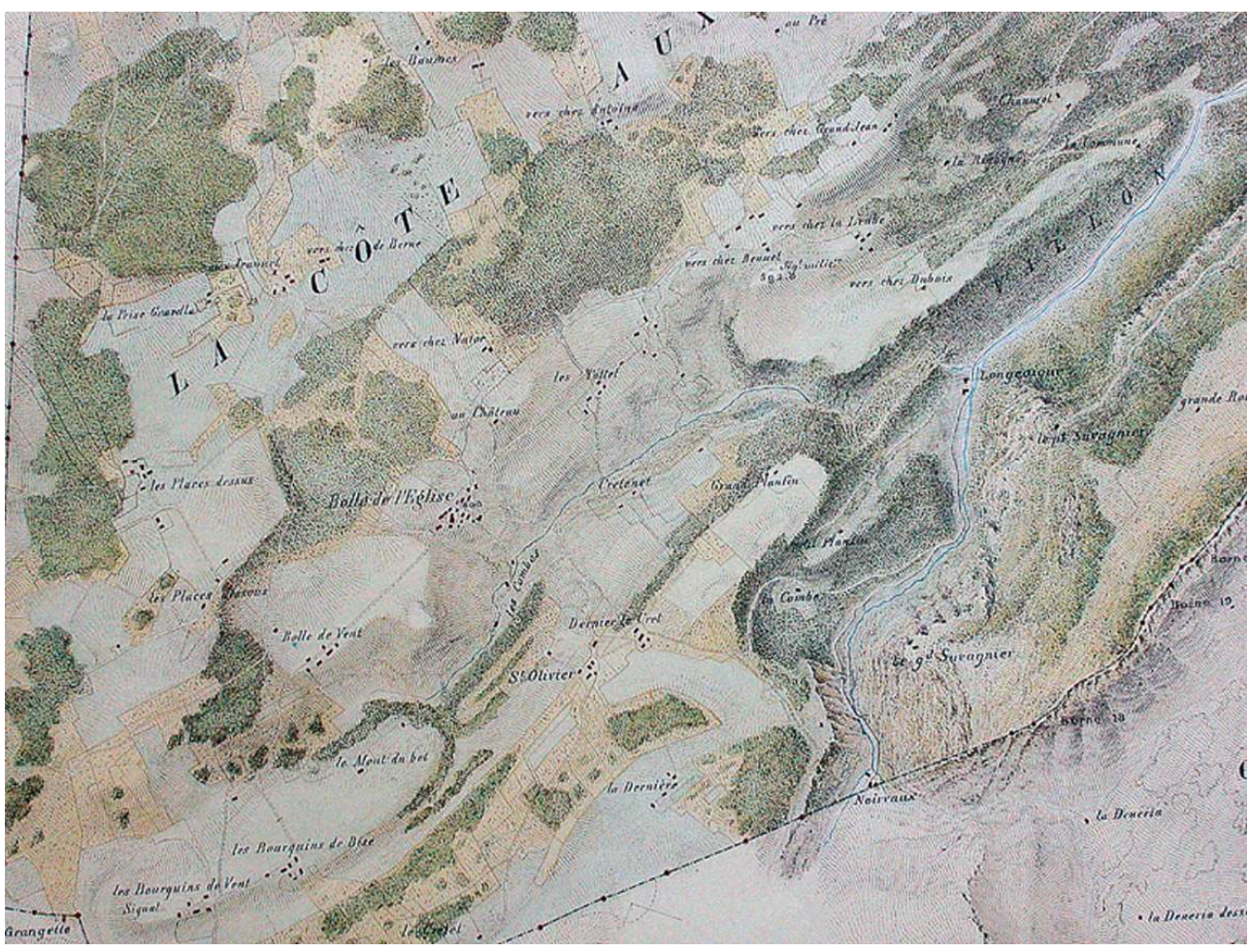

Dispersion en hameaux sur le territoire de la Côte-aux-Fées selon la carte Osterwald de 1838-1845 conservée aux Archives cantonales neuchâteloises

Phot. D. Glauser (c) D. Glauser 
Figure 24

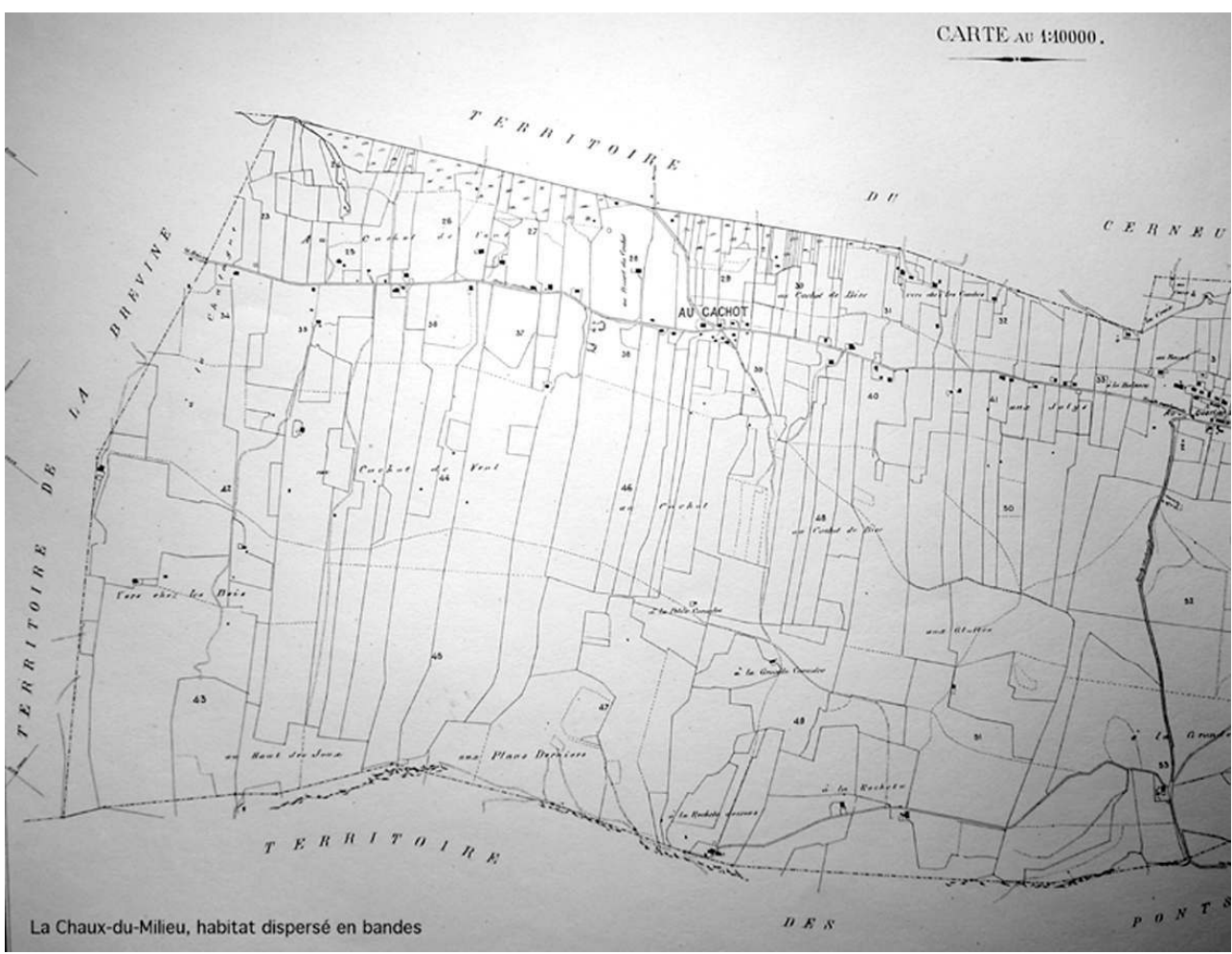

Dispersion en bandes à la Chaux-du-Milieu selon une carte conservée aux Archives cantonales neuchâteloises

Phot. D. Glauser @ D. Glauser 
Figure 25

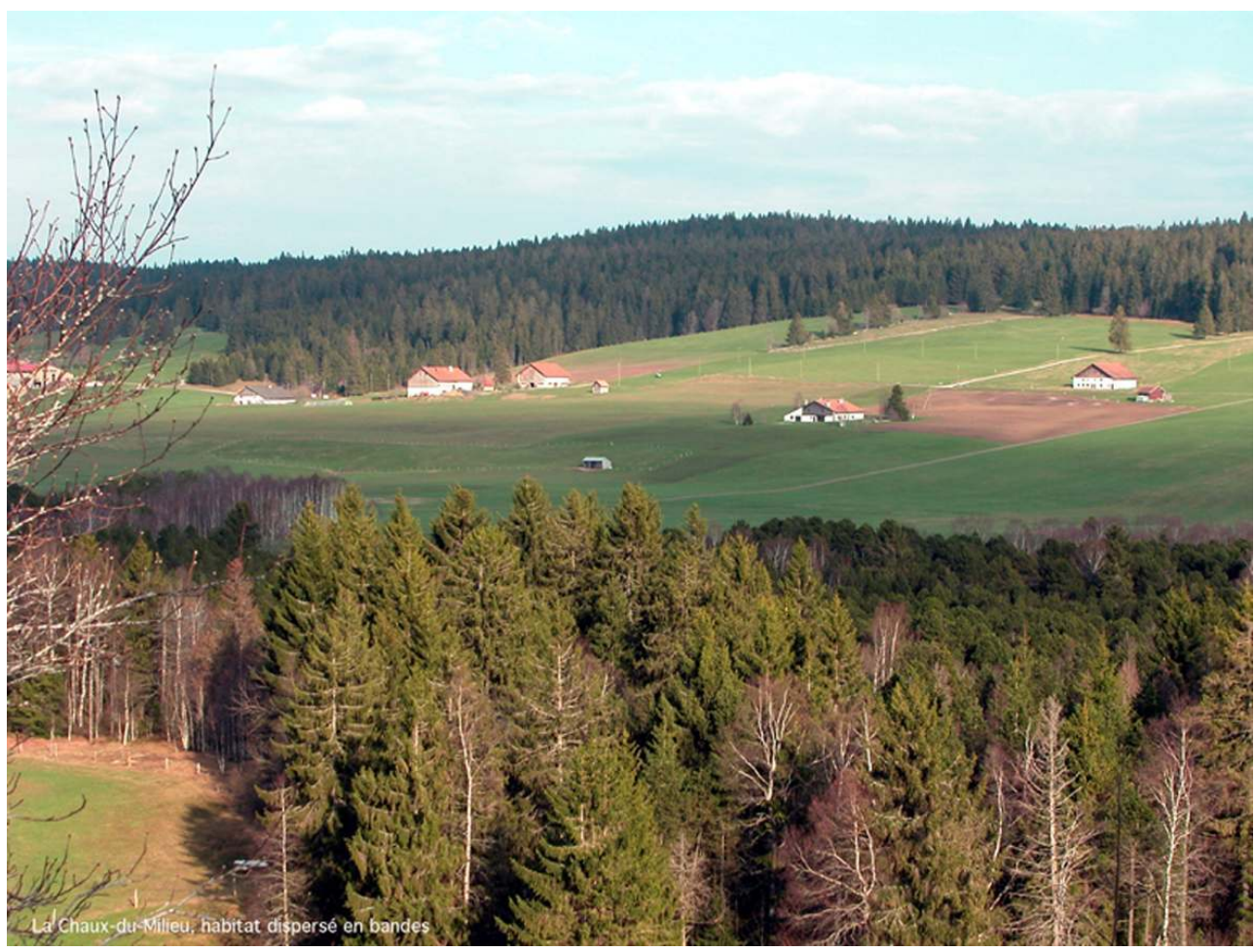

Dispersion en bandes à la Chaux-du-Milieu

Phot. D. Glauser ( ) D. Glauser

\section{BIBLIOGRAPHIE}

Anderegg, Jean-Pierre. La maison paysanne fribourgeoise, Broye, Glâne, Gruyère et Veveyse. Bâle : Les maisons rurales de Suisse, tome 8, 1987.

Anderegg, Jean-Pierre. La maison paysanne fribourgeoise, die Bezirke Saane, See, Sense. Bâle : Les maisons rurales de Suisse, tome 7, 1979.

Biermann, Charles. La maison paysanne vaudoise. Lausanne, 1946.

Egloff, Wilhelm, Egloff-Bodmer, Anne-Marie. Les maisons rurales du Valais. Le pays, la construction en bois, la maison d'habitation. Bâle : Les maisons rurales de Suisse, tome 13, 1987.

Flückiger-Seiler, Roland, Constantin, Luc. Les Maisons rurales du Valais, tome 14. Bâle, 2000.

Glauser, Daniel. Les maisons rurales du canton de Vaud. Tome 1. Le Jura vaudois et ses contreforts. Bâle : Les maisons rurales de Suisse, tome 16, 1989.

Glauser, Daniel. Les maisons rurales du canton de Vaud. Tome 3. De La Côte à la Venoge, tome 18. Bâle, 2002. 
Glauser, Daniel. Les maisons rurales du canton de Vaud. Tome 4. Du Gros-de-Vaud à la Broye, tome 19. Bâle, 2003.

Raymond, Denyse. Les maisons rurales du canton de Vaud. Préalpes, Chablais, Lavaux. Bâle : Les maisons rurales de Suisse, tome 17, 2002.

\section{NOTES}

1. Daniel Glauser est employé par l'Etude des maisons rurales de Suisse, il exerce actuellement son activité principale au Service des monuments et des sites du canton de Neuchâtel; il collabore à l'établissement de l'inventaire du patrimoine rural et à la rédaction des ouvrages consacrés aux maisons rurales de ce canton.

2. Lebeau, René. Les grands types de structure agraire dans le monde. Paris, 1979, p. 32.

\section{RÉSUMÉS}

En Suisse romande, la topographie marquée du paysage allant de la chaîne jurassienne à celle des Alpes, ainsi que l'histoire du peuplement, ont contribué à générer une grande variété de formes de colonisation. Dans les cantons de Vaud et de Neuchâtel notamment, on observe aussi bien du paysage de bocage que des territoires marqués par l'assolement triennal collectif avec des exploitations agricoles en "champs ouverts" (openfield). Les villages sont organisés selon une grande variété de schémas, avec des structures linéaires horizontales, montantes ou en épi, des formes rayonnantes sur un point ou sur un anneau. La dispersion de l'habitat rural obéit également à différents modèles : petits hameaux, par unité d'exploitation avec une répartition alvéolaire ou en bandes des domaines.

In French-speaking Switzerland, the characteristic topography of the landscape, between the mountain ranges of the Jura and the Alps, along with the history of how these landscapes were peopled, have given rise to a wide variety of forms of settlement. In the cantons of Vaud and Neufchatel in particular, there are landscapes of mixed pasture and woodland (bocage) as well as territories characterised by the collective three-field system with open-field farms. The villages too are structured according to a broad variety of patterns: horizontal linear structures, structures going up the slope with branches going off at angles, structures with radial patterns leading off from a central point or from a ring. Dispersed rural dwellings also follow different models: small hamlets with individual farms or with organisations in honeycomb patterns or bands of property. 


\section{INDEX}

Mots-clés : Suisse, Suisse romande, canton de Vaud, canton de Neuchâtel, bocage, paysage, assolement, triennal, openfield, village, habitat rural, hameau, Jura vaudois, agriculture de montagne, Gros-de-Vaud, Broye, Echallens, unité d'exploitation, Val-de-Ruz, village aggloméré, habitat dispersé, La Comballaz, Lavaux, Ormonts-Dessous, Le Creux-du-Van, Mutrux, Joux-duPlâne, Vaulion, Burtigny, Penthalaz, Prahins, Epalinges, Chaux-du-Milieu

Keywords : Switzerland, French-speaking Switzerland, canton of Vaud, canton of Neufchatel, bocage, landscape, three-field system, openfield, village, rural dwellings, hamlets, Vaudois Jura, mountain agriculture, Gros-de-Vaud, Broye, Echallens, farm, Val-de-Ruz, agglomerated village, dispersed habitat, La Comballaz, Lavaux, Ormonts-Dessous, Le Creux-du-Van, Mutrux, Joux-dePlâne, Vaulion, Burtigny, Penthalaz, Prahins, Epalinges, Chaux-du-Milieu

\section{AUTEUR}

\section{DANIEL GLAUSER}

Ethno-géographe. daniel.glauser@ne.ch 